\title{
Green Technology Adoption and the Business Cycle
}

\author{
Jean-Marc Bourgeon and Margot Hovsepian*
}

April 16, 2018

\begin{abstract}
We analyze the adoption of green technology in a dynamic economy affected by random shocks where demand spillovers are the main driver of technological improvements. Firms' beliefs about the best technology and consumers' anticipation drive the path of the economy. We derive the optimal policy of investment subsidy and the expected time and likelihood of reaching a targeted level of environmental quality under economic uncertainty. This allows us to estimate the value as a function of the strength of spillover effects that should be given to the environment in order to avoid an environmental catastrophe.
\end{abstract}

Keywords: Growth, sustainability, uncertainty.

JEL: O33, O44, E37, Q55

* Bourgeon: INRA, UMR économie Publique, 16 rue Claude Bernard, 75231 Paris Cedex 05, France, and Department of Economics, Ecole Polytechnique, France (e-mail: bourgeon@agroparistech.fr); Hovsepian: Department of Economics, Ecole Polytechnique, 91128 Palaiseau Cedex, France. 
"I believe that ultimately the electric motor will be universally used for trucking in all large cities, and that the electric automobile will be the family carriage of the future. All trucking must come to electricity. I am convinced that it will not be long before all the trucking in New York City will be electric."

Thomas Edison, Automobile Topics, May 1914.

\section{Introduction}

The increasing number of environmental issues that the world is facing has triggered a wide debate on how to switch to sustainable development paths. Adoption of green technologies (AGT) is one of the main channels through which countries will be able to avoid environmental disasters without overly harming their well-being. However, adopting new, cleaner technologies is risky for firms. At first, they incur a switching cost: green technologies are often more expensive and less productive, and the workforce may not have the skills to operate the new technology. Moreover, in the long run, investment choices may turn out to be inefficient, harming the firms' profitability ${ }^{1}$ Hence, even if a technology is available (at the turn of the 20th century, 38\% of American automobiles were powered by electricity) and is endorsed by even such a preeminent inventor and businessman as Thomas Edison, that does not guarantee that it is the best choice to be made. Both network externalities and technological spillovers play an important role in determining what is the optimal technology for firms to adopt. Public policies may be designed to help firms to overcome this risk and to invest in green technologies, but the extent of this investment effort also depends on the prevailing economic environment. If the economy is facing a recession, available funds may be scarce, leading firms to postpone investment projects. We may thus expect the path toward environmental sustainability to be stochastically affected by the same economic shocks as those that generate the business cycle.

In this paper, we analyze this problem using a simple AGT model, focusing on an environmental policy that takes the form of subsidizing investment in green technologies. We determine the relationship between the volatility in the adoption path of technology and the value that should be given to the environmental quality (EQ)

\footnotetext{
${ }^{1}$ Consider for example that a firm decides to replace its fleet of fuel vehicles by electric ones (EVs). If many firms expect EVs to be used nationwide in the near future and decide to do the same, it is likely that the network of EV charging stations will spread broadly and using EVs may become very convenient and cheap. If instead the rest of the economy turns to hydrogen vehicles, then the few firms that have chosen EVs may end up being penalized.
} 
in order to avoid an environmental disaster. We consider a setting where industrial production continuously harms the environment. By investing in less-polluting technologies, firms contribute to lowering their impact on the environment. Firms' profit depends on their past and present investment choices that are subsumed in a "technology mix" index, a parameter that measures the pollution intensity of their production process. A key feature of our framework is that a firm being equipped at a given date with the most profitable production process does not mean that its machines are the most recent or innovative ones. Instead, the profitability of the various production processes depends also on technological spillovers like the skills of the workforce that use the technology, and the availability of the inputs and of the maintenance services required by the technology. The more a technology is used, the better the diffusion of knowledge and the networks of related services, including the research effort of the machine industry sector, implying that the "optimal technology mix" depends on the investment choices made by all firms. Each period, firms devote a certain amount of resources to conform their technology to the most efficient one. To decide on their investment, they form expectations based on private and public information and may undershoot or overshoot the optimal technology index. The imperfect assessment firms have about their future economic environment results in an industrial sector composed of firms with heterogeneous technological processes. This explains why along any equilibrium path different technologies coexist at the same time, some more largely adopted than others.

Because markets do not account for the environmental footprint of the economy, the social planner designs a policy that redirects the firms' technology indexes toward greener mixes. The policy implemented by the social planner internalizes the marginal benefit of AGT and thus of a better EQ on social welfare. There is often substantial scientific uncertainty about the impact of human activities on environmental resilience (the capacity of ecosystems to respond to shocks). It is generally considered that the environment has an intrinsic ability to partly regenerate and recover from past damages as long as EQ has not dropped below a tipping point, i.e. a possible threshold for abrupt changes, from one stable state to another, catastrophic one, after a transition period that may be irreversible $2^{2}$ One of the public objectives is thus to avoid EQ crossing the tipping point and wandering around hazardous levels. However, since business cycles

\footnotetext{
${ }^{2}$ In climatology, several tipping points have been given, e.g. the Amazon rainforest dieback, the loss of Polar ice packs and melting of Greenland and Antarctic ice sheets, the disruption of Indian and West African monsoons, the loss of permafrost. The IPCC Fifth Assessment Report states that precise levels remain uncertain.
} 
and uncertainty affecting AGT make the path of the economy stochastic, the economy may at some point hit or even pass the tipping point while transitioning toward a desired long-term value.

Our model captures these various uncertainties in a very parsimonious way thanks to two convenient analytical tools commonly used in financial economics: global games and chance-constrained stochastic programming $]^{3}$ The firms' problem of adapting their production process over time is framed as a dynamic global game where a continuum of firms, characterized at date $t$ by their current technological mix, faces a coordination problem guided by private and public signals about the next period's optimal technology mix. We focus on Markov perfect equilibria where firms' optimal investment strategy is linear and signals are normally distributed. The social planner's problem is expressed as a chance-constrained stochastic program: the difference between the level of EQ and the tipping point can be compared to a "budget" (in the case of the global warming problem that we consider in our numerical simulations, a "carbon" budget) that, in order to avoid being at risk of bankruptcy, society should not exhaust. As the path of the economy is stochastic, the social planner must find the optimal policy such that this budget is not exhausted with a certain level of confidence. Depending on the value attached by individuals to the environment, this constraint could be binding over a long period, i.e., securing only a minimum carbon budget for decades could be optimal if the individuals' direct utility from the carbon budget is low. However, just as a financial firm that manages its portfolio of assets using the value-at-risk criterion may, because of unexpected large shocks, go bankrupt nevertheless, society still faces the risk of an environmental catastrophe even while maintaining EQ at the chance-constrained level 4 A relevant benchmark is thus the minimal value that should be given to the environment so that this budget constraint along the optimal path is never binding: this Chance-Constrained Value of the Environment (CCVE) can serve as the Social Cost of Carbon (SCC) in the case of the global warming problem ${ }^{5}$

\footnotetext{
${ }^{3}$ Global games are used in the literature on crises in financial markets (see Morris \& Shin, 2003 for an overview). More generally, they allow us to model the problem of aggregating information in markets where agents have imperfect information on economic fundamentals and whose payoffs are interdependent. The "Value at Risk" criterion in portfolio selection is an instance of chanceconstrained stochastic programming (see, e.g., Shapiro et al., 2009 for an extensive discussion of stochastic programming methods).

${ }^{4}$ Even before the Industrial Revolution and the current debate on sustainable carbon dioxide concentrations in the atmosphere, the world climate went through some dramatic changes, like the socalled "Little Ice Age" of the 16th century, or the "Great Frost" of 1709 which is believed to have caused 600,000 deaths in France.

${ }^{5}$ In the Integrated Assessment Model literature, the SSC corresponds to the sum of the marginal welfare losses (evaluated along the optimal policy path) caused by the immediate release of an ad-
} 
We exhibit a framework where the average technological mix and the other state variables of the economy follow a Gaussian random walk that permits estimation of confidence intervals for the realized paths of AGT and EQ indexes. The optimal subsidy policy is characterized through benchmark values of the interest rate that should prevail at equilibrium on the financial market. The targeted rate is high at the beginning of the public intervention: firms benefit from large subsidies to improve their technology mix, which leads to a high demand of capital. The average interest rate is higher the higher the marginal value of the environment. It stays at this level as long as the economy traverses a transition period that is eventually followed by a carbon-neutral trajectory, i.e. a situation where the industrial sector no longer impacts the environment. Along this path, the targeted rate decreases toward its stationary value and so does the public subsidy.

We illustrate this approach with numerical simulations that show a positive relationship between the technological spillovers and the CCVE. The more spillover effects are at work, the more incentives should be given to direct firms' choices toward a sustainable (carbon-neutral) path, but also the fastest this aim is reached under the optimal policy. The major impact of technological spillovers on the optimal policy comes from their impact on catastrophic risk: a large subsidy is required because the risk of reaching the environmental tipping point is high.

Our model is related to the large literature on growth and sustainability. The endogenous green growth literature focuses on productivity improvements and frontier innovation. This is the case in the AK paradigm where capital-knowledge accumulates with learning-by-doing (Stockey, 1998), in Lucas-like extensions (Bovenberg \& Smulders, 1995), in a product variety framework (Gerlagh \& Kuik, 2007) or in the Schumpetarian growth paradigm of destructive creation and directed technical changes (Acemoglu et al., 2012), where the most productive innovations are adopted by firms as soon as they are discovered. While frontier innovation is needed to make green technologies competitive compared to "brown" ones, our focus is on adoption of existing technologies that go with spillover effects causing a gradual replacement of old and polluting machines by greener ones. Our approach is thus close to the literature on endogenous growth viewed as a process of adoption of existing ideas and mutual imitation between firms, as exposed by Eaton \& Kortum (1999); Lucas Jr \& Moll (2014); Lucas

ditional ton of carbon dioxide emissions that create lasting reductions of output through the rise in temperatures (see, e.g. Nordhaus, 2014). We consider instead (arguably equivalently) that in addition to consumption, individuals enjoy having a good EQ but their willingness to pay for it is not related to the risks faced by future generations. 
(2009); Perla \& Tonetti (2014). In these papers, it is assumed that each agent in the economy is endowed with a certain amount of knowledge ("ideas") and this knowledge evolves through contact with the rest of the population. We adopt a similar approach to describe AGT: while there is no explicit R\&D sector in our model, there is a pool of existing technologies with potentials that are more or less exploited depending on the proportion of firms that use them. The distribution of technology used among firms shifts over time according to the firms' incentives to adopt new techniques.

Most of the literature on sustainable growth focuses on environmental uncertainty, that is, uncertainty on the frequency of catastrophic environmental events, on the size of the damage, or on the ability of the environment to recover from pollution 6 Contrary to these analyses, we suppose that the hazard rate function (which links the likelihood of the catastrophe to EQ) and the extent of the damages on social welfare caused by the catastrophe are unknown. The social planner knows only that doomsday is more likely the lower the EQ, particularly once this index has reached the tipping point. Its objective is thus to maximize the expected intertemporal utility, avoiding EQ passing the environmental threshold. The few papers that describe the responsiveness of optimal abatement policy to business cycles (Jensen \& Traeger, 2014) or that assess the optimal policy instrument in stochastic environments (Heutel, 2012, Fischer \& Heutel, 2013) do not consider AGT. In the few integrated assessment models encompassing economic risk such as Golosov et al. (2014) or Traeger (2015), there is no absorbing lower bound in the dynamics of the environmental quality, and even though there is a risk that the environmental policy is not optimal given the realized shocks, there will be no irreversible consequences. Our model instead gives us a simple tool to derive the SCC such that given the optimal green technology subsidy, EQ avoids reaching a tipping point with a large enough probability.

The remainder of the paper is organized as follows: In section 2 , we describe the economy and the dynamics of the AGT index. In section 3, we discuss the social planner's program and we characterize the optimal policy of investment subsidy. In section 4, we specify this policy in a framework that allows the economy to follow a Gaussian random path. Section 5 is devoted to numerical simulations. The last section concludes.

\footnotetext{
${ }^{6}$ See, e.g. Tsur \& Zemel $(\sqrt{1998})$ or De Zeeuw \& Zemel $(2012)$ for analysis without AGT, and Bretschger \& Vinogradova (2014) and Soretz (2007) for studies on the sharing of investment between productive capital and abatement measures in an AK setting.
} 


\section{Green technology dynamics}

The economy is composed of a continuum of firms, of total mass equal to one, that collectively produce at date $t$ an amount $q_{t}$ of output, taken as the numeraire, which corresponds to the GDP of the economy. In the following, we abstract from the problem of production per se (in particular, the demand and supply of labor) to focus on the cleanliness of the production processes, i.e. the extent to which firms harm the environment while producing. We take an agnostic stance on the relative productivity of 'brown' and 'green' technologies by supposing that the GDP follows the same dynamic whatever the economy's technological mix of productive capital, and more specifically that it is given by the following first-order autoregressive dynamic

$$
q_{t}=g q_{t-1}+\hat{g}+\kappa_{t}
$$

with $1>g \geq 0, \hat{g} \geq 0$, and where $\kappa_{t}$ corresponds to exogenous shocks that affect the economy at date $t$ and is the realization of random variable $\left.\tilde{\kappa}_{t} \sim \mathcal{N}\left(0, \sigma_{\kappa}^{2}\right)\right]^{7}$ As $g<1$, the per-period expected increase in GDP, $\hat{g}-(1-g) q_{t-1}$, diminishes over time and converges to $q_{S}=\hat{g} /(1-g) \cdot 8$ The production processes used by firms are diverse and their environmental impacts are captured by real value parameters dubbed 'technology mixes' or 'green technology' indexes, denoted $x_{i t}$ for firm $i$ at date $t$, which on average lead to an AGT index of the industrial sector given by $\mu_{t} \equiv \int x_{i t} d i$. Each period, firm $i$ may decide to spend $I_{i t}$ to improve its technology mix that evolves according to

$$
x_{i t+1}=x_{i t}+I_{i t}
$$

The spendings (or savings) $I_{i t}$ comes in addition to the capital necessary to increase the firm's productivity; its corresponds to outlays on productive investments that are due to the buying of technologies greener or browner than the ones embedded in $x_{i t}$. With green technologies costlier than brown ones, a positive $I_{i t}$ indicates that firm $i$ is greening its production process. These spendings allow firms to adapt their mix to the economic environment that will prevail the next period. A mix different from the optimal one is costly because of the specific knowledge and skills required to operate and maintain technologies that are not widely used or because of the relative scarcity

\footnotetext{
${ }^{7}$ Throughout the paper, a random variable is topped with a tilde symbol $(\sim)$ to distinguish it from its possible values.

${ }^{8}$ The case $g>1$ corresponds to a steady growth in economic wealth. Sustainable states in that case are not stationary: the economy should instead follow a sustainable balanced path.
} 
of inputs employed. Firms would ideally be equipped with the most efficient mix, but it evolves with the diffusion of knowledge and the know-how of workers (the so-called knowledge spillover: the more firms invest, the better the workers' knowledge of new technologies in general), network externality (how easy it is to find specific inputs and parts to service the technology) and the engineering and research effort exerted by the machine industry firms that compete to satisfy the demand for means of production in the economy. To grasp these various effects in a parsimonious way, we suppose that firm $i$ 's ideal mix at time $t$ is given by

$$
x_{i t}^{\star}=\lambda_{i t} \mu_{t-1}+\left(1-\lambda_{i t}\right) \mu_{t}
$$

where $\lambda_{i t}$ corresponds to firm's $i$ characteristics at that time (i.e. the composition of its local economic environment, from its network of suppliers to the know-how of its employees). $\lambda_{i t}>1$ corresponds to a firm at the front edge of the spectrum, because of a favorable local environment, while $\lambda_{i t}<1$ denotes a backward situation. Since the actual technology mix $x_{i t}$ results from investment choices made in previous periods, the firm $i$ 's date- $t$ revenue net of the spending to adapt its mix for the next period is given by

$$
\pi\left(x_{i t}, I_{i t} ; x_{i t}^{\star}\right)=\Pi\left(x_{i t}^{\star}\right)-\left(x_{i t}^{\star}-x_{i t}\right)^{2} / 2-I_{i t}
$$

where $\Pi\left(x_{i t}^{\star}\right)$ is the firm's potential revenue and $\left(x_{i t}^{\star}-x_{i t}\right)^{2} / 2$ the loss due to a less effective mix than $x_{i t}^{\star}$. As firms' local environments evolve (due, e.g. to new hires and choices made by other firms around), we suppose that the firms' specificities of the next period, $\lambda_{i t+1}$, are unknown to their managers at the time they make their investment choices and correspond to idiosyncratic and time-independent draws from the same time-invariant distribution. Denoting by $\mathcal{I}_{i t}$ the information of firm $i$ at time $t$, we thus have $\mathbb{E}_{t}\left[\tilde{\lambda}_{i t+1} \mid \mathcal{I}_{i t}\right]=\lambda$ for all $i$ and $t$. The next period optimal index of the industrial sector is given by

$$
x_{t+1}^{\star}=\int x_{i t+1}^{\star} d i=\lambda \mu_{t}+(1-\lambda) \mu_{t+1}=\mu_{t}+\lambda \int I_{i t} d i
$$

where $\lambda$ captures the green technology demand spillover. We assume that $\lambda$ is positive but lower than 1 because of the slow spreading of new technologies $9^{9}$ If $\lambda=0$, the average best mix for the next period corresponds to the current average mix $\mu_{t}$. When demand spillovers are at work $(\lambda>0)$, it also depends on the investment choices made

\footnotetext{
${ }^{9}$ As stressed by Battisti (2008), a consistent literature has shown that, even when a clean or a cost-reducing technology is ready available in the market, its spreading takes several years.
} 
by all firms, which creates a coordination problem. As firms make their investment decisions simultaneously each period, they must somehow anticipate the extent of the resulting total investment. This intertemporal coordination problem is formalized as a succession of global games. ${ }^{10}$ Each period, firms form their expectations on $x_{i t+1}^{\star}$ thanks to a public signal and firms' private (idiosyncratic) signals on the average optimal index, $\tilde{\omega}_{t}=x_{t+1}^{\star}+\tilde{\eta}_{t}$, and $\tilde{w}_{i t}=x_{t+1}^{\star}+\tilde{\varepsilon}_{i t}$ respectively, where $\tilde{\eta}_{t}$ and $\tilde{\varepsilon}_{i t}$ are normally distributed time-independent noises with zero mean and precision $\tau_{\eta}=\sigma_{\eta}^{-2}$ and $\tau_{\varepsilon}=$ $\sigma_{\varepsilon}^{-2}$ verifying $\mathbb{E}\left[\tilde{\varepsilon}_{i t} \tilde{\varepsilon}_{j t}\right]=0$ for all $i, j$ and $\int \tilde{\varepsilon}_{i t} d i=0$. These signals allow firms to (imperfectly) coordinate their investment levels each period although their decisions are taken independently. This dynamic setup is solved sequentially: our focus is on Markov perfect equilibria where the economic fundamentals $x_{t}^{\star}$ is a state variable that firms must anticipate each period to decide on their investment levels. More specifically, Bayesian updating implies that firm $i$ 's posterior beliefs about $x_{t+1}^{\star}$ given its signals are normally distributed with mean

$$
\hat{x}_{i t+1} \equiv \mathbb{E}\left[\tilde{x}_{t+1}^{\star} \mid \omega_{t}, w_{i t}\right]=\frac{\tau_{\eta} \omega_{t}+\tau_{\varepsilon} w_{i t}}{\tau_{\eta}+\tau_{\varepsilon}}=x_{t+1}^{\star}+\tau \eta_{t}+(1-\tau) \varepsilon_{i t},
$$

where $\tau=\tau_{\eta} /\left(\tau_{\eta}+\tau_{\varepsilon}\right)$ is the relative precision of the public signal, and variance

$$
\hat{\sigma}_{i t}^{2}=\left(\tau_{\eta}+\tau_{\varepsilon}\right)^{-1}
$$

for all $i$ and $t$. The firm chooses its investment plan to maximize the discounted sum of per-period profit (4), which is equivalent to minimizing the expected sum of the discounted revenue loss compared with the optimal mix. Applying the principle of optimality, the firm's optimal plan is derived by solving the Bellman equation

$$
\mathcal{V}\left(x_{i t} ; x_{i t}^{\star}\right)=\min _{I_{i t}}\left(x_{i t}-x_{i t}^{\star}\right)^{2} / 2+I_{i t}+\delta_{t} \mathbb{E}_{t}\left[\mathcal{V}\left(x_{i t}+I_{i t} ; \tilde{x}_{i t+1}^{\star}\right) \mid \omega_{t}, w_{i t}\right]
$$

where $\delta_{t}$ is the discount factor corresponding to interest rate $r_{t}$, i.e. $\delta_{t}=\left(1+r_{t}\right)^{-1}$. It is shown in the appendix that

\footnotetext{
${ }^{10}$ These coordination problems with strategic complementarity are known as "beauty contests" (see, e.g., Angeletos \& Pavan, 2004, 2007 and Morris \& Shin, 2002). Compared to this literature focusing on the learning of some exogeneous economic fundamentals that influence the firms' investment strategies, we suppose instead that the economic fundamental to be anticipated by the firms is precisely the result of their investments.
} 
Proposition 1 Firm i's equilibrium investment strategy at time $t$ is given by

$$
I_{i t}=\hat{x}_{i t+1}-x_{i t}-r_{t}
$$

The firms' technology levels at $t+1$ are normally distributed with mean

$$
\mu_{t+1}=\mu_{t}-\left(r_{t}-\tau \eta_{t}\right) /(1-\lambda)
$$

corresponding to the date- $t+1 A G T$ level of the economy, and variance

$$
\sigma_{x}^{2} \equiv(1-\tau)^{2} \sigma_{\varepsilon}^{2}
$$

According to (9), firms' investment strategy is to adapt their production process to their estimate of the average most efficient mix diminished by the price of capital $r_{t}$, which leads firms to disinvest. For firms with a low mix, this strategy corresponds to buying more environmentally friendly equipment. For the others, their investment is directed in the opposite direction: they can save on new equipment spending by buying less expensive brown technologies. On average, this heterogeneity in investment policies should somehow be canceled out, but while this is true for idiosyncratic noises, the public signal $\eta_{t}$ distorts firms choices in the same direction to an extent that depends on its reliability $\tau$ : the better the signal's precision, the larger the distortion. Indeed, given the firms' investment strategy

$$
I\left(\omega_{t}, w_{j t}, x_{j t}, r_{t}\right)=\tau \omega_{t}+(1-\tau) w_{j t}-r_{t}-x_{j t}
$$

we obtain that on average, as $\int \varepsilon_{j t} d j=0$,

$$
\int I\left(\omega_{t}, \tilde{w}_{j t}, x_{j t}, r_{t}\right) d j=x_{t+1}^{*}+\tau \eta_{t}-r_{t}-\mu_{t},
$$

and thus that at a rational expectation equilibrium, the optimal average mix satisfies

$$
x_{t+1}^{*}=\mu_{t}+\lambda\left[x_{t+1}^{*}+\tau \eta_{t}-r_{t}-\mu_{t}\right]=\mu_{t}+\lambda\left(\tau \eta_{t}-r_{t}\right) /(1-\lambda) .
$$

The shock $\eta_{t}$ also modifies the dynamic of the AGT index given by 100 which is derived from (12) using (5). This shock is magnified by a factor equal to $(1-\lambda)^{-1}$ : the larger $\lambda$, the larger the effects of the public signal and of the cost of capital $r_{t}$. Without governmental intervention, as $\mathbb{E}\left[\mu_{t+1}\right]=\mu_{t}-r_{t} /(1-\lambda)$, the larger $\lambda$, the lower the AGT 
index. Indeed, as investment is costly in the absence of an environmental policy, each firm anticipates that other firms' investments will be low, which leads to an equilibrium that tends to the lower bound of the AGT index.

Due to the idiosyncratic shocks on believes, $\varepsilon_{i t}$, firms have different expectations on $x_{t+1}^{\star}$. These discrepancies lead to a Gaussian distribution of firms' green indexes around the AGT level with a dispersion that is larger the better the relative precision of the idiosyncratic shocks $1-\tau$. Hence, the industrial sector can be thought of as a 'cloud' of firms with a technology level that is drawn each period from a normal distribution centered on the AGT index $\mu_{t}$ with standard deviation $\sigma_{x}$.

To counteract the negative effect of the interest rate on green investment, we assume in the following that the government implements at date $t_{0}$ a subsidy policy plan $\left\{z_{t}\right\}_{t \geq t_{0}}$ leading to a per-period investment cost $r_{t}-z_{t}$. The dynamic of the AGT index (10) then becomes

$$
\mu_{t+1}=\mu_{t}+\left(z_{t}-r_{t}+\tau \eta_{t}\right) /(1-\lambda)
$$

which increases in expectation if $z_{t}>r_{t}$, i.e. if the net cost of capital is negative.

\section{Consumers}

Consumers maximize their intertemporal utility by arbitraging between consumption and savings every period. Their well-being mainly comes from consumption, but it is also impacted by the environment, either from private awareness and concerns for environmental issues, or because of tangible consequences of climate change such as damages due to more frequent extreme weather events. More precisely, we consider that their per-period preferences on consumption $c$ and environmental quality $e$ are represented by a utility function $u_{\phi}(c, e)$ increasing in $c$ and $e$ and concave, that belongs to a parametric family where $\phi$ indexes the consumers' marginal rate of substitution (MRS) of consumption for EQ:

$$
\frac{d}{d \phi}\left[\frac{\partial u_{\phi}(c, e) / \partial e}{\partial u_{\phi}(c, e) / \partial c}\right]>0
$$

We suppose that all consumers are identical and thus that $\phi$ characterizes society as a whole. We also suppose that, while aware of the impact of EQ on their welfare, consumers do not try to modify the environment through their consumption and saving plans. This could be the case because they consider that they are too numerous for 
their individual behavior to have a significant impact on the environmental path 11 Accordingly, we model their behavior by considering a representative consumer whose saving and consumption plans solve

$$
\max \mathbb{E}_{t}\left\{\sum_{\tau=t}^{+\infty} \beta^{\tau-t} u_{\phi}\left(\tilde{c}_{\tau}, \tilde{e}_{\tau}\right): \tilde{c}_{\tau}=\tilde{R}_{\tau}+\tilde{r}_{\tau-1} \tilde{S}_{\tau-1}-\tilde{s}_{\tau}, \tilde{s}_{\tau}=\tilde{S}_{\tau}-\tilde{S}_{\tau-1}\right\}
$$

where $R_{t}$ is her date- $t$ revenue, $S_{t-1}$ her savings from the previous period, $r_{t-1} S_{t-1}$ the corresponding date- $t$ capital earnings, $s_{t}$ the savings adjustment of period $t$ and $\beta$ the psychological discount factor. Solving the consumer's program, we obtain

Lemma 1 The consumption rule that solves (14) satisfies

$$
\partial u_{\phi}\left(c_{t}, e_{t}\right) / \partial c=\left(1+r_{t}\right) \beta \mathbb{E}_{t}\left[\partial u_{\phi}\left(\tilde{c}_{t+1}, \tilde{e}_{t+1}\right) / \partial c\right]
$$

at each date $t$.

Equation (15) corresponds to the Ramsey-Euler rule in our setup. It also defines the supply function of capital, while (13) defines the demand side coming from firms. At the date- $t$ equilibrium on the capital market, the interest rates embodied in (15) and (13) are equal. Moreover, at the goods market equilibrium, aggregate production net of investment must be equal to total consumption, i.e. ${ }^{12}$

$$
c_{t}=q_{t}-\int I_{i t} d i=q_{t}-\mu_{t+1}+\mu_{t}
$$

Together, these two conditions allow us to determine the global equilibrium that the economy reaches each period.

\section{The environment}

Production generates pollution, which harms the environment, but this detrimental effect can be reduced if firms improve their technology parameter. This mechanism is embodied in the following dynamic of the EQ index

$$
e_{t+1}=\theta e_{t}+\hat{e}-\iota_{t} q_{t}
$$

\footnotetext{
${ }^{11}$ The environmental quality being a public good, this reasoning is grounded by the standard freerider argument that results in the underprovision of public goods.

${ }^{12}$ Observe that the firm's relative loss $\left(x_{i t}-x_{t}^{\star}\right)^{2} / 2$ does not appear in 16 since it corresponds to expenses (e.g. payroll outlays, external services) that are revenues for other agents in the economy.
} 
where $0<\theta<1, \hat{e} \geq 0$ and where $\iota_{t}$ is the emission intensity of the technology mix at date $t$, which measures the damage to the environment coming from human activities per unit of GDP. $\hat{e}$ is the per-period maximum regeneration capacity of the environment, the actual regeneration level reached at period $t$ being $\hat{e}-(1-$ $\theta) e_{t-1}$, which depends (positively) on the environmental inertia rate $\theta$. Without human interference $\left(\iota_{t}=0\right)$, the EQ index is at its pristine level $e_{N}=\hat{e} /(1-\theta)$. Emission intensity is related to the AGT index by

$$
\iota_{t}=\varphi-\xi \mu_{t} / q_{t}
$$

where $\varphi$ is the maximum emission intensity and $\xi>\sqrt{(1-\theta) \varphi}$ so that green technologies are sufficiently effective. Substituting for $\iota_{t}$ in (17), we obtain a dynamic of EQ that follows the linear first-order recursive equation

$$
e_{t+1}=\theta e_{t}+\xi \mu_{t}-\varphi q_{t}+\hat{e}
$$

We suppose that $\varphi q_{S}>\hat{e}$, implying that without AGT, the environment will collapse once production is sufficiently large and will eventually reach the "tipping point" $\bar{e}$ that should not be passed permanently: if pollution is too high too often, the resilience of the environment is at stake, i.e. abrupt shifts in ecosystems may happen with dire and irreversible consequences for society ${ }^{13}$ On the other hand,

Definition 1 (Environmentally Neutral Path) The economy has reached at date $T$ an Environmentally Neutral Path (ENP) if for all $t \geq T, \mathbb{E}\left[\tilde{\iota}_{t}\right]=0$.

Therefore, an ENP is a sustainable situation in which the expected emission intensity of the economy is nill. The AGT subsidy that is required once the economy has reached an ENP should allow for $\mu_{t}$ to stay at $\varphi q_{t} / \xi$ in expectations. ENPs are of most interest because we consider green technologies that aim to reducing emissions (i.e. they do not allow for direct improvement of EQ) and thus environmental neutrality is the best society can achieve. Along an ENP, thanks to the natural regeneration

\footnotetext{
${ }^{13}$ There is often substantial scientific uncertainty about the resilience of ecosystems and thus a debate about the relevant value of these environmental thresholds: drastic changes may happen when EQ is larger than a given referential $\bar{e}$ (or nothing at all while EQ is lower), suggesting that the date of the catastrophic event, $\mathcal{T}$, depends on EQ stochastically. Formally, this corresponds to a survival function at time $t, \mathcal{S}_{t}=\operatorname{Pr}\left\{\mathcal{T}>t \mid e_{t}\right\}$ that increases with $e_{t}$, but the lack of scientific knowledge prevents us from specifying it. The debate over $\bar{e}$ is thus about the "reasonable" maximum exposure of society to a potential catastrophic collapse, but since there is always such a risk, a cautious strategy is to make sure that EQ is as large as possible.
} 
capacity of the environment, the average EQ increases and tends toward its pristine level $e_{N}$.

However, reaching an ENP may prove to be too demanding and society may end up stabilizing around an expected EQ level $e_{S}<e_{N}$. This sustainable situation corresponds to the notion of a stable environment path:

Definition 2 (Stable Environment Path) The economy has reached at date $T$ a Stable Environment Path (SEP) at level $e_{S}$ if for all $t \geq T, \mathbb{E}\left[\tilde{\iota}_{t} \tilde{q}_{t}\right]=(1-\theta)\left(e_{N}-e_{S}\right)$.

Under an SEP, technological improvements must fill the gap between the environmental damages due to economic growth and the regenerative capacity of the environment to maintain EQ at the desired level over time. Compared to its ENP levels, the expected AGT index is lower by a constant proportional to the difference between the pristine EQ level $e_{N}$ and the stabilized one $e_{S}:(1-\theta)\left(e_{N}-e_{S}\right)$ corresponds to the per-period loss of EQ compared to the pristine level that society does not compensate for. Nevertheless, to stay at a constant EQ level, the AGT index must increase at the same pace as the emissions coming from aggregate production.

\section{Environmental policy}

We consider a benevolent social planner who decides on a policy that modifies the dynamics of the AGT level (13) through setting $z_{t}$ for all $t \geq t_{0}$ where $t_{0}$ is the first period where the policy is designed and implemented. The aim of the policy is twofold: first, it has to correct for the fact that the consumer's value of the environment is not reflected in the interest rate that prevails at equilibrium on the capital market. Second, while the MRS at the citizen level allows individuals to link EQ with the damages they encounter, these evaluations are likely to be underestimations of the actual contribution of EQ to global welfare. Indeed, a measure of the social value of EQ should encompass all the services provided by the environment and, in particular, the fact that the lower EQ, the more at risk the resilience of the environment is and thus the larger the risk of a catastrophe for future generations. Hence, since there is always a non-zero probability that the actual environmental path hits $\bar{e}$ under the optimal policy, the social planner must also ensure that an environmental disaster is avoided with at least a certain level of confidence, i.e. that $\operatorname{Pr}\left\{\tilde{e}_{t} \leq \bar{e}\right\} \leq \alpha$ for all $t \geq t_{0}$, where $\alpha$ corresponds to the 
chosen confidence level. The chance-constrained problem that she must solve is thus ${ }^{14}$

$$
\max \left\{\mathbb{E}_{t_{0}} \sum_{t=t_{0}}^{+\infty} \beta^{t-t_{0}} u_{\phi}\left(\tilde{c}_{t}, \tilde{e}_{t}\right): \mathbb{E}\left[\tilde{\iota}_{t}\right] \geq 0, \operatorname{Pr}\left\{\tilde{e}_{t} \leq \bar{e}\right\} \leq \alpha\right\}
$$

where the first constraint corresponds to the ENP constraint (emission intensity cannot be negative) and the second to the Environmental Safety (ES) constraint ${ }^{15}$ The public policy affects the dynamic of the economy in the following way. From (13), the path of the AGT index evolves stochastically according to

$$
\tilde{\mu}_{t+1}=\mu_{t}+\left(z_{t}-\tilde{r}_{t}+\tau \tilde{\eta}\right) /(1-\lambda)
$$

In addition to directly modifying the AGT path, it also indirectly affects the equilibrium on the goods market since we have, from (22) and (16),

$$
\tilde{c}_{t}=q_{t}-\left(z_{t}-\tilde{r}_{t}+\tau \tilde{\eta}\right) /(1-\lambda) \text {. }
$$

Finally, as we suppose that the social planner does not intervene directly in the capital market, the interest rate at equilibrium must satisfy the Euler equation

$$
\mathbb{E}\left[\partial u_{\phi}\left(\tilde{c}_{t}, e_{t}\right) / \partial c\right]=\beta \mathbb{E}\left[\left(1+\tilde{r}_{t}\right) \partial u_{\phi}\left(\tilde{c}_{t+1}, \tilde{e}_{t+1}\right) / \partial c\right]
$$

which is the ex ante equivalent of $(15)$. Consider the problem of the social planner at date $t$ when the state of the economy is far from both the ENP and ES constraints.

\footnotetext{
${ }^{14}$ The term "chance-constrained programming" was first coined by Charnes \& Cooper (1959). Yaari (1965) provides an early comparison of this approach with the penalty/loss function procedure in a lifetime expected utility maximization problem.

${ }^{15}$ Alternatively, we may consider an intertemporal state-dependent utility setup (see e.g., Tsur \& Zemel 2008, 2009; Bommier et al. 2015, propose an Epstein-Zin recursive utility framework). This can be done by assuming, e.g., that the random date of the catastrophe $\mathcal{T}$ follows an exponential distribution that depends on EQ so that the survival function at time $t$ is given by $\mathcal{S}_{t}=\operatorname{Pr}\{\mathcal{T}>$ $\left.t \mid e_{t}\right\}=1-e^{-p e_{t}}$. The public objective then becomes

$$
\mathbb{E}_{t_{0}} \sum_{t=t_{0}}^{+\infty} \beta^{t-t_{0}}\left[\left(1-e^{-p \tilde{e}_{t}}\right) u\left(\tilde{c}_{t}, \tilde{e}_{t}\right)+e^{-p \tilde{e} \tilde{e}_{t}} \underline{u}\right]
$$

where $\underline{u}$ is the doomsday continuation utility. Compared to the chance-constraint approach, this setup requires to know the relevant values of $p$ and $\underline{u}$, and more generally, the hazard rate of the catastrophe as a function of EQ and its impacts on humanity, which is beyond the current level of scientific knowledge.
} 
The corresponding Bellman equation is given by

$$
\left.\mathcal{W}\left(\mu_{t}, e_{t}, q_{t}\right)=\max _{z_{t}}\left\{\mathbb{E}_{t}\left[u_{\phi}\left(\tilde{c}_{t}, e_{t}\right)\right]+\beta \mathbb{E}_{t}\left[\mathcal{W}\left(\tilde{\mu}_{t+1}, \tilde{e}_{t+1}, \tilde{q}_{t+1}\right)\right]:(1),(19),(22),(23), 24\right)\right\}
$$

where $\mu_{t}, e_{t}$ and $q_{t}$ are state variables and $z_{t}$ the control variable. It is shown in the appendix that:

Proposition 2 The production/environment state that solves (25) satisfies

$$
r_{t+1}^{e}+r_{t}^{e} r_{t+1}^{e}-r_{t}^{e} \theta=\xi \mathbb{E}\left[\partial u_{\phi}\left(\tilde{c}_{t+2}, \tilde{e}_{t+2}\right) / \partial e\right] / \mathbb{E}\left[\partial u_{\phi}\left(\tilde{c}_{t+2}, \tilde{e}_{t+2}\right) / \partial c\right]
$$

where

$$
r_{t}^{e} \equiv \frac{\mathbb{E}\left[\tilde{r}_{t} \partial u_{\phi}\left(\tilde{c}_{t+1}, \tilde{e}_{t+1}\right) / \partial c\right]}{\mathbb{E}\left[\partial u_{\phi}\left(\tilde{c}_{t+1}, \tilde{e}_{t+1}\right) / \partial c\right]}=\mathbb{E}\left[\tilde{r}_{t}^{\star}\right]+\frac{\operatorname{cov}\left(\tilde{r}_{t}, \partial u_{\phi}\left(\tilde{c}_{t+1}, \tilde{e}_{t+1}\right) / \partial c\right)}{\mathbb{E}\left[\partial u_{\phi}\left(\tilde{c}_{t+1}, \tilde{e}_{t+1}\right) / \partial c\right]}
$$

The unconstrained dynamic of the economy is thus determined through the sequence of the "corrected" expected optimal interest rate $r_{t}^{e}$ that solves (26). Compared to $\mathbb{E}\left[\tilde{r}_{t}^{\star}\right]$, it takes into account the (positive) correlation between the marginal utility of consumption and the interest rate, as shown in (27).

If the consumer's valuation of EQ is very low (i.e., $\phi$ low), it could be the case that the ES constraint is binding at some date $T$ and that the regulated economy follows an SEP during a long period at expected level $e_{S}>\bar{e}$, the edge depending on $\alpha$. Or, for larger $\phi$, that this constraint is only temporarily binding while the regulated economy is on its way toward an ENP. To gauge the value of the environment along a stochastic path of the economy, we consider the discounted MRS of consumption for EQ along this path as expected at time $t_{0}$, i.e.

$$
\rho_{\phi}=(1-\beta) \mathbb{E}_{t_{0}} \sum_{t=t_{0}}^{+\infty} \beta^{t-t_{0}} \frac{\partial u_{\phi}\left(\tilde{c}_{t}, \tilde{e}_{t}\right) / \partial e}{\partial u_{\phi}\left(\tilde{c}_{t}, \tilde{e}_{t}\right) / \partial c}
$$

which increases with $\phi$. A benchmark for the public policy is given by

Definition 3 The chance-constrained value of the environment (CCVE) is the lowest value of the discounted MRS evaluated along the optimal expected path of the economy such that the ES constraint is never binding: it is given by $\rho_{\phi^{*}}$ where

$$
\phi^{*}=\arg \min _{\phi}\left\{\max \left\{\mathbb{E}_{t_{0}} \sum_{t=t_{0}}^{+\infty} \beta^{t-t_{0}} u_{\phi}\left(\tilde{c}_{t}, \tilde{e}_{t}\right): \mathbb{E}\left[\tilde{\iota}_{t}\right] \geq 0\right\}: \operatorname{Pr}\left\{\tilde{e}_{t} \leq \bar{e}\right\}<\alpha\right\}
$$


The CCVE $\rho_{\phi^{*}}$ corresponds to hypothetical preferences $\phi^{*}$ that separate two types of societies: those with $\phi>\phi^{*}$, for which the SE constraint is not binding, and those with $\phi<\phi^{*}$ which are more at risk of being struck by a catastrophe in some distant future. It is an interesting benchmark because, as the path of the economy of any society depends on $\lambda$, this edge is affected by the technological spillovers.

Because we consider a public objective that integrates a safety constraint that could be binding, the social planner may be considered "paternalistic", i.e. imposing a route to individuals for their own sake. This is even more so if it considers that the CCVE must inform the policy because of the selfishness of individuals regarding future generations and the lack of scientific knowledge that makes it difficult to thoroughly evaluate the risk that those future generations face. In this regard, the SE constraint is a minimum safety measure, and avoiding its binding corresponds to a sensible safeguard.

\section{The constant MRS - CARA utility case}

In the rest of the article, we derive the optimal policy assuming that the representative consumer's preferences are CARA and that consumption and environmental quality can be subsumed in a 'global wealth index' denoted $y_{t} \equiv c_{t}+\rho e_{t}$, so that the MRS is the same whatever the GDP of the economy (we thus have $\phi=\rho=\rho_{\phi}$ in that case). 16 Under theses assumptions, the dynamic of the economy follows a Gaussian random walk and the optimal policy is easily characterized 17

With a constant MRS, (26) simplifies to

$$
r_{t+1}^{e}+r_{t}^{e} r_{t+1}^{e}-r_{t}^{e} \theta=\rho \xi
$$

${ }^{16} \mathrm{~A}$ constant MRS is a reasonable approximation as long as $g<1$ (i.e. wealth is bounded by $q_{S}$ ) and the environment has not incurred dramatic changes $\left(e_{t}\right.$ is above $\left.\bar{e}\right)$. Assuming steady growth $(g>1)$, as the environmental quality is bounded upward, this price should increase at the same pace as GDP along the balanced growth path: $\rho_{t}=g^{t} \rho_{0}$.

${ }^{17}$ Interestingly, under these assumptions the problem of finding the CCVE may be written as

$$
\min _{p} \max \mathbb{E}_{t_{0}}\left\{\sum_{t=t_{0}}^{+\infty} \beta^{t-t_{0}} e^{-p \tilde{e}_{t}} u\left(\tilde{c}_{t}\right): \mathbb{E}\left[\tilde{\iota}_{t}\right] \geq 0, \operatorname{Pr}\left\{\tilde{e}_{t} \leq \bar{e}\right\}<\alpha\right\}
$$

which can be interpreted as the expected sum of losses in consumption well-being: compared to (21) in footnote 15 , only the last sum appears with the continuation utility $\underline{u}$ replaced by $u\left(\tilde{c}_{t}\right)$, the utility of consumption of the period. Increasing $p$ diminishes the per-period probability of the catastrophe and thus of the total losses: the larger $p$, the more optimistic is the viewpoint. Hence, by considering the lowest $p$ such that the environmental safety constraint is not binding, the social planner adopts a careful standpoint (the minimax regret criterion). The difference between programs (20) and 21) is particularly apparent in the case where $\underline{u}=0$ in the latter: then, the social planner only considers the well-being of generations that have avoided the catastrophe. 
which prescribes the evolution of the interest rate from one period to the next along an optimal path when neither the ENP constraint nor the ES constraint are binding. Observe that this dynamic does not depend on the state of the economy (none of the state variables $q_{t}, e_{t}$ and $\mu_{t}$ is involved). From its initial value $r_{t_{0}}^{e}$, it may converge to a long run level that solves

$$
\left(r_{\sharp}^{e}+1-\theta\right) r_{\sharp}^{e}=\rho \xi \text {. }
$$

The left-hand side of $(29)$ is a quadratic equation which is positive for either $r_{\sharp}^{e} \leq$ $-(1-\theta)$ or $r_{\sharp}^{e} \geq 0$, while the right-hand side is strictly positive. Hence $r_{\sharp}^{e}$ may take two values, one being positive and the other negative and lower than $-(1-\theta)$. Fig. 1 depicts the situation.

[Figure 1 about here.]

The parabola corresponds to the left-hand side of (29) which crosses the x-axis at 0 and $-(1-\theta)$. The horizontal line corresponds to the right-hand side of (29). The negative root of (29) is lower than $-(1-\theta)$, and increasingly so the larger $\rho \xi$. As $r_{t}^{e}$ is greater than the optimal expected interest rate that must prevail in the long term on the capital market, only the positive root of 29 is relevant.

The following proposition describes the transition of the economy toward this longterm interest rate.

Proposition 3 With a constant $M R S$, the solution of (28) is given by

$$
r_{t}^{e}=r_{\sharp}^{e}+\frac{A\left(r_{t_{0}}^{e}-r_{\sharp}^{e}\right) k^{t-t_{0}}}{A+\left(1-k^{t-t_{0}}\right)\left(r_{t_{0}}^{e}-r_{\sharp}^{e}\right)}
$$

for all $t \geq t_{0}$, where $A=\sqrt{(1-\theta)^{2}+4 \rho \xi}$ is the square root of the discriminant of (29),

$$
r_{\sharp}^{e}=(A-1+\theta) / 2
$$

the positive root of (29), and

$$
k=(1+\theta-A) /(1+\theta+A) .
$$

$r_{t}^{e}$ converges to $r_{\sharp}^{e}$ if $\rho \xi \neq \theta$ and $r_{t_{0}}^{e}>-(A+1-\theta) / 2$. Convergence is monotonic if $\rho \xi<\theta$ and oscillatory if $\rho \xi>\theta$.

The sequence of rates given by (30) allows the social planner to estimate at time $t_{0}$ the expected path of the interest rate that should prevail on the capital market using 
(40) and thus to decide on the policy measure $z_{t}$ to be implemented. It depends on an initial value $r_{t_{0}}^{e}$ deduced from the current state of the economy that should not be too negative. This sequence converges to (31) which depends on the social value of the environment $\rho$ and on the parameters that govern the dynamic of the environment: the environmental inertia $\theta$ and the parameter that captures the impact of the technology index on the environment $\xi$. It should be noted that Prop. 3 describes the dynamic of the economy when neither the ENP constraint nor the ES one is binding.

To detail the convergence of the interest rate given by (30), it is convenient to use the normalized gap between the expected interest rate at horizon $h$ and its long run level which is defined as

$$
d_{t_{0}+h} \equiv\left(r_{t_{0}+h}^{e}-r_{\sharp}^{e}\right) / A=\left(\mathbb{E}\left[r_{t_{0}+h}^{\star}\right]-r_{\sharp}^{\star}\right) / A .
$$

It is shown in the appendix that

Proposition 4 With a constant $M R S$, the normalized gap at horizon $h$ from $t_{0}$ can be derived recursively using $d_{t_{0}+h}=f\left(d_{t_{0}+h-1}\right)$ where

$$
f(x) \equiv k x /[1+(1-k) x]
$$

with $k$ defined by (32) in Prop. 3. This gap converges to 0 provided $d_{t_{0}}>-1$.

A phase diagram of this recursion is given fig. 2 which depicts the relationship $d_{t+1}=f\left(d_{t}\right)$ in the $\left(d_{t}, d_{t+1}\right)$ plane and where the bisector is used as a "mirror" to project the value $d_{t+1}$ back on the $d_{t}$ axis.

[Figure 2 about here.]

Function $f(\cdot)$ is concave, with a vertical asymptote at $d_{t}=-1 /(1-k)$ and an horizontal one for large values of $d_{t}$. We have $f(0)=0$ and $f^{\prime}(0)=k<1$, hence the bisector is located above the graph of function $f(\cdot)$ in the positive quadrant and since $f(-1)=-1$, below it in the negative quadrant for all $d_{t} \leq-1$. From an initial gap $d_{t_{0}}$ the arrows describe the recursion toward 0 which occurs for all $d_{t_{0}}>-1$.

Applying recursively $d_{t_{0}+h}=f\left(d_{t_{0}+h-1}\right)$ gives the expected value of the optimal interest rate from one period to the next and thus allows the government to define the entire policy at time $t_{0}$. To initiate the recursion, we suppose that the government is able to determine the expected value of the interest rate at date $t_{0}-1$, the period before the policy is implemented, which allows it to derive the initial normalized gap: 
$d_{t_{0}-1}=\left(r_{t_{0}-1}-r_{\sharp}^{\star}\right) / A$. The normalized gap of the first period is deduced from this value using $d_{t_{0}}=f\left(d_{t_{0}-1}\right)$. The first period expected optimal interest rate is deduced from $\mathbb{E}_{t_{0}}\left[\tilde{r}_{t_{0}}^{\star}\right]=r_{\sharp}^{\star}+A d_{t_{0}}$, and the sequence of all future ones from applying $\mathbb{E}_{t_{0}}\left[\tilde{r}_{t}^{\star}\right]=r_{\sharp}^{\star}+A d_{t}$ with $d_{t}=f\left(d_{t-1}\right)$ for all $t>t_{0}$. This allows us to analyze the commitment (also dubbed open-loop) strategy that supposes that the government sticks to this plan whatever the state of the economy in the subsequent periods.18

To complete the analysis, we derive the Rational Expectation Equilibrium (REE) of this economy assuming that the consumer's preferences over global wealth are CARA, i.e. that we have $u_{\phi}\left(c_{t}, e_{t}\right)=-e^{-\gamma\left(c_{t}+\rho e_{t}\right)}$ where $\gamma$ corresponds to the coefficient of absolute risk aversion. If the global wealth index $y_{t+1}$ is normally distributed (which is the case at equilibrium), we get from 15 using $\mathbb{E}\left[e^{-\gamma \tilde{y}}\right]=e^{-\gamma(\mathbb{E}[\tilde{y}]-\gamma \mathbb{V}[\tilde{y}] / 2)}, \beta=e^{-\psi}$ where $\psi$ is the intrinsic discount factor, and $1+r_{t} \approx e^{r_{t}}$, a supply function of capital satisfying 19

$$
r_{t}=\psi+\gamma\left(\mathbb{E}_{t}\left[\tilde{y}_{t+1}\right]-y_{t}\right)-\gamma^{2} \mathbb{V}_{t}\left[\tilde{y}_{t+1}\right] / 2
$$

which exhibits the familiar effects that determine the rental price of capital: the intrinsic preference for an immediate consumption $\psi$, the economic trend of the global wealth index that also encourages immediate consumption if it is positive, and as revealed by the last term, a precautionary effect that operates in the opposite direction and corresponds to a risk premium due to the uncertainty affecting the economy. Given the environmental policy that will be implemented by the government $\left\{z_{t+h}\right\}_{h \geq 0}$, we obtain that

Proposition 5 Under an REE with preferences over global wealth and CARA utility, the dynamic of the technological parameter can be approximated by

$$
\mu_{t+1}=a_{1} \mu_{t}+a_{2} e_{t}+a_{3} q_{t}+a_{4}+a_{5} \tau \eta_{t}+Z_{t}
$$

where

$$
Z_{t}=a_{5} \sum_{i=0}^{+\infty}\left(\gamma a_{5}\right)^{i} z_{t+i}
$$

and the distribution of the wealth index $\tilde{y}_{t+1}$ by a normal distribution with variance

$$
\sigma_{y}^{2} \equiv \mathbb{V}_{t}\left[\tilde{y}_{t+1}\right]=\left(1-a_{3}\right)^{2} \sigma_{\kappa}^{2}+a_{5}^{2} \tau^{2} \sigma_{\eta}^{2}
$$

\footnotetext{
${ }^{18}$ Note that $(34)$ can also be used as a tool to update the environmental policy each period taking as initial value the observed current gap.

${ }^{19}$ While 35 is derived using the approximation $1+r_{t} \approx e^{r_{t}}$, it should be noted that the discrepancy between the exact formula for the interest rate and 35 is only an artifact of the discrete time setup: the shorter the time period, the better the approximation.
} 
leading to stationary value of the interest rate $r_{S} \equiv \psi-\gamma^{2} \sigma_{y}^{2} / 2$. Moreover, we have $a_{1}<1$ and $a_{1}>0$ if $2+(1-\lambda) / \gamma-\theta>\rho \xi, 0<a_{2}<\rho, a_{3}>0,0<a_{5}<1 / \gamma$ and $a_{4}<0$ whenever $r_{S} \geq 0, d a_{1} / d \lambda<0, d a_{2} / d \lambda>0, d a_{3} / d \lambda>0$ and $d a_{5} / d \lambda>0$.

The equilibrium dynamic of the AGT index follows a linear first-order recursive equation of the state variables $\left(\mu_{t}, e_{t}, q_{t}\right)$ and of a forward looking term $Z_{t}$ given by (37) that accounts for the anticipated effects of the environmental policy. This policy index is an exponential smoothing of the policy measures to come. This dynamic is thus consistent only if the policy plan is known. Assuming that $\rho$ is not too large, all coefficients are positive except the constant $a_{4}$ which is negative $\left(r_{S} \geq 0\right.$ is a sufficient condition only for $a_{4}$ to be negative) ${ }^{20}$ Hence, one may thus expect that under laissezfaire, even if the AGT and EQ indexes have reached very low levels, the increase in the GDP could reverse a negative trend: this is indeed the case when $q_{t}>-a_{4} / a_{3}$ (assuming that a catastrophe is avoided). As expected, the stronger the spillover effects captured by $\lambda$, the less the dynamic of the AGT index is dependent on its previous value and the more it is on the GDP and the economic shocks. But as $a_{5}$ determines the exponential smoothing coefficient of $Z_{t}$, the dynamic is also more reactive to the public policy when $\lambda$ is large.

Because of the linearity of (36), the AGT index $\tilde{\mu}_{t+2}$ as estimated at date $t$ is Gaussian (while the date- $t$ realizations of the shocks are known, $\tilde{\mu}_{t+2}$ depends on the next period shocks $\tilde{\kappa}_{t+1}$ and $\tilde{\eta}_{t+1}$ ). From (16), $\tilde{c}_{t+1}$ is thus normally distributed, resulting in global wealth index $\tilde{y}_{t+1}$ which is also Gaussian with variance given by (38). Knowing $\left\{z_{t+h}\right\}_{h \geq 0}$, it is possible to infer statistically the state of the economy at horizon $h$ from an initial state at date $t$ by applying recursively (36) together with (1), (19), thanks to the normal distribution of the random shocks.

Using (13) or (35) and (36), it is possible at the beginning of each period to specify the distributions of the equilibrium interest rate. More precisely, we have

Lemma 2 Under an REE with preferences over global wealth and CARA utility, before the current period shock and signals are known, the current interest rate and the next period wealth index are normally distributed. Their variances are given by

$$
\sigma_{r}^{2}=(1-\lambda)^{2} a_{3}^{2} \sigma_{\kappa}^{2}+\left[1-(1-\lambda) a_{5}\right]^{2} \tau^{2} \sigma_{\eta}^{2}
$$

\footnotetext{
${ }^{20}$ If $\rho$ is very large, coefficient $a_{1}$ is negative which generates oscillations in the AGT dynamic (36). We suppose that it is not the case and thus that fluctuations in the AGT index are only the consequences of the shocks affecting the economy.
} 
and

$$
\sigma_{y_{+1}}^{2}=\sigma_{y}^{2}+\left[\left(1-a_{3}\right) g+\left(1-a_{1}\right) a_{3}+\left(a_{2}-\rho\right) \varphi\right]^{2} \sigma_{\kappa}^{2}+\left[\left(1-a_{1}\right) a_{5} \tau \sigma_{\eta}\right]^{2}
$$

respectively. The expected interest rate corresponding to the optimal policy (27) is given by

$$
\mathbb{E}_{t}\left[\tilde{r}_{t}^{\star}\right]=r_{t}^{e}-(1-\lambda) a_{3} \gamma\left(\sigma_{y_{+1}}^{2}-\sigma_{y}^{2}\right)+\gamma\left[1-(1-\lambda)\left(a_{5}-a_{3}\right)\right]\left(1-a_{1}\right)^{2} a_{5}^{2} \tau^{2} \sigma_{\eta}^{2}
$$

Observe that the variance of the interest rate is different from $\gamma^{2} \sigma_{y}^{2}$. This is due to the fact that the two bracketed terms in (35) are correlated random variables when the current shocks are unknown: the expectation of $\tilde{y}_{t+1}$ without knowing the realization of $\tilde{y}_{t}$ is a random variable. Similarly, the variance of the next period wealth index differs form $\sigma_{y}^{2}$ by terms accounting for the correlation between $\tilde{y}_{t+1}$ and $\tilde{y}_{t}$. Finally, we obtain that

Proposition 6 The economy follows an ENP or an SEP reached at date $T$ if the government implements the policy $\left\{z_{t}\right\}_{t \geq T}$ given by

$$
z_{t}=R_{t}+(1-\lambda)(1-g) g^{t-T}\left(q_{S}-q_{T}\right) \varphi / \xi
$$

where

$$
R_{t}=r_{S}+\gamma(1-g) g^{t-T}\left(q_{S}-q_{T}\right)[1+(1-g) \varphi / \xi]+\gamma \rho(1-\theta) \theta^{t-T}\left(e_{S}-e_{T}\right)
$$

converges toward $r_{S}$ as $t$ goes to infinity, with $e_{T}=e_{S}<e_{N}$ under an SEP and $e_{T}<e_{S}=e_{N}$ under an ENP. Under an REE with preferences over global wealth and CARA utility, the corresponding policy index $\left\{Z_{t}\right\}_{t \geq T}$ is given by

$$
\begin{aligned}
Z_{t} & =\frac{a_{5} r_{S}}{1-a_{5} \gamma}+\frac{(1-g) g^{t-T}\left(q_{S}-q_{T}\right)[\gamma+(1-\lambda+\gamma(1-g)) \varphi / \xi]}{1-a_{5} \gamma g} \\
& +\frac{\gamma \rho(1-\theta) \theta^{t-T}\left(e_{S}-e_{T}\right)}{1-a_{5} \gamma g \theta} .
\end{aligned}
$$

In case of an SEP, denoting by $\Phi$ the CDF of the standardized Gaussian variable, $e_{S}=\bar{e}+\sigma_{e} \Phi^{-1}(1-\alpha)$ where $\sigma_{e}$ is independent of the initial state of the economy.

Sequence $\left\{R_{t}\right\}_{t \geq T}$ given by 42 corresponds to the path of the expected interest rate $\mathbb{E}\left[\tilde{r}_{t}\right]$ along an ENP or an SEP. From (41), it follows that in both cases the gap 
between the governmental subsidy and the expected interest rate shrinks exponentially. The expected interest rate also diminishes steadily toward its stationary state value $r_{S}$ (which should not be confused with the long-run level $r_{\sharp}^{\star}$ toward which the expected interest rate converges during the transition phase). The last term of (42) which involves the difference between the state of the environment at date $T$ and its long term level, is positive in the case of an ENP since the environment keeps improving, while it is equal to zero for an SEP since, by definition, the environment is stabilized at level $e_{S}<e_{N}$.

\section{Policy implementation and Environmental forecast}

Assuming that the social planner implements a policy that results in a sequence of expected interest rates $\mathbb{E}_{t_{0}}\left[\tilde{r}_{t}^{\star}\right]$ given by 40 , eventually followed by a sequence satisfying (42) once the economy has reached either an SEP or an ENP, the expected paths of the AGT and EQ indexes can be anticipated using the system of equations (1), (19) and 36 which gives the recursion

$$
\tilde{Y}_{t}=B_{t} \tilde{Y}_{t-1}+H \tilde{\nu}_{t}
$$

where $\tilde{Y}_{t}=\left(\tilde{\mu}_{t}, \tilde{e}_{t}, \tilde{q}_{t}, 1\right)^{\prime}$ is the column vector of state values (with the constant), $B_{t}$ is the time-dependent transition matrix

$$
B_{t}=\left[\begin{array}{cccc}
a_{1} & a_{2} & a_{3} & a_{4}+Z_{t} \\
\xi & \theta & -\varphi & \hat{e} \\
0 & 0 & g & \hat{g} \\
0 & 0 & 0 & 1
\end{array}\right], H=\left[\begin{array}{cc}
a_{5} \tau \sigma_{\eta} & 0 \\
0 & 0 \\
0 & \sigma_{\kappa} \\
0 & 0
\end{array}\right]
$$

and $\tilde{\nu}_{t}=\left(\tilde{\nu}_{1 t}, \tilde{\nu}_{2 t}\right)^{\prime}$ is a column vector of independent standardized Gaussian variables. The transition matrix $B_{t}$ is time-dependent because of the policy index $Z_{t}$ defined by (37) which is an exponential smoothing of the policy measures that will be implemented in the following periods. This index is given by (43) after the economy has reached either an SEP or a ENP. For the transitory period where the state of the economy is not constrained, using (13) taken in expectation at date $t_{0}$, we obtain that the optimal subsidy scheme satisfies

$$
z_{t+i}=\mathbb{E}\left[\tilde{r}_{t+i}^{\star}\right]+(1-\lambda)\left(\mathbb{E}\left[\tilde{\mu}_{t+i+1}\right]-\mathbb{E}\left[\tilde{\mu}_{t+i}\right]\right) .
$$


Multiplying each side of 45 by $a_{5}\left(\gamma a_{5}\right)^{i}$ and summing over all $i \geq 0$ gives

$$
\begin{aligned}
Z_{t} & =a_{5} \sum_{i=0}^{T-t-1}\left(\gamma a_{5}\right)^{i} \mathbb{E}\left[\tilde{r}_{t+i}^{\star}\right]+a_{5} \sum_{i=T-t}^{+\infty}\left(\gamma a_{5}\right)^{i} R_{t+i} \\
& +a_{5}(1-\lambda)\left(1-\gamma a_{5}\right) \sum_{i=0}^{+\infty}\left(\gamma a_{5}\right)^{i}\left(\mathbb{E}\left[\tilde{\mu}_{t+i+1}\right]-\mathbb{E}\left[\tilde{\mu}_{t+i}\right]\right)
\end{aligned}
$$

for all $t<T$. Because the last term of (46) involves values of the AGT index that depend on the public policy, it is not possible to obtain the optimal public policy schedule explicitly and a numerical (recursive) procedure is necessary. The recursion proceeds as follows: given a first set of values $\left\{\mathbb{E}\left[\tilde{\mu}_{t}\right]^{(1)}\right\}_{t \geq t_{0}}$, it is possible to estimate $\left\{Z_{t}^{(1)}\right\}_{t \geq t_{0}}$ from 46 to obtain an initial set of transition matrices $\left\{B_{t}^{(1)}\right\}_{t \geq t_{0}}{ }^{21}$ Then, using 44 , a second set of estimated values $\left\{\mathbb{E}\left[\tilde{\mu}_{t}\right]^{(2)}\right\}_{t \geq t_{0}}$ is derived which, once plugged in 46), gives a second estimated set $\left\{Z_{t}^{(2)}\right\}_{t \geq t_{0}}$ and thus $\left\{B_{t}^{(2)}\right\}_{t \geq t_{0}}$. One may iterate this procedure until iteration $i$ such that $\left\{Z_{t}^{(i)}\right\}_{t \geq t_{0}}$ is sufficiently close to $\left\{Z_{t}^{(i-1)}\right\}_{t \geq t_{0}}$.

As the recursive dynamic (44) is linear, the path it generates follow Gaussian random walks with expected values and variances at time $t$ given by $\mathbb{E}\left[\tilde{Y}_{t}\right]=\left(\Pi_{i=0}^{t-t_{0}} B_{i}\right) X_{t_{0}}$ and $\mathbb{V}\left[\tilde{Y}_{t_{0}}\right]=\sum_{i=0}^{t-t_{0}}\left(\Pi_{j=0}^{i} B_{j}\right) H H^{\prime}\left(\Pi_{j=0}^{i} B_{j}\right)^{\prime}$.

\section{$5 \quad$ Numerical simulations}

In this section, we illustrate the impact of the technological spillovers on the optimal path of the economy in the case of the global warming problem. The expected trends of the main economic variables and the confidence intervals in which they are lying can be derived under various assumptions using numerical simulations 22 Of particular interest is the relationship between the technological spillovers and the CCVE (SEPs are not considered) given a confidence level 23

\section{Calibration of the model}

In the following, EQ is defined as the difference between a tipping point level of $\mathrm{CO}_{2}$ in the atmosphere $\ell_{M}$ and the level of GHG at date $t, \ell_{t}$, expressed in $\mathrm{CO}_{2}$ equivalent:

\footnotetext{
${ }^{21}$ Of course, date $T$ at which one of the constraints is binding should be derived in the process.

${ }^{22}$ These simulations were realized using Mathematica. The corresponding programs are available from the authors upon request.

${ }^{23}$ The CCVE in this context could be compared to the SCC. While different from the usual interpretation of the SCC, it is in the spirit of approaches that account for catastrophic damages (see, e.g., Weitzman, 2013).
} 
$e_{t}=\ell_{M}-\ell_{t}{ }^{24}$ Hence, $e_{t}$ can be thought of as a global "carbon budget" at date $t$ that the economy should not deplete entirely to avoid to be environmentally bankrupt. The CCVE value $\rho^{*}$ corresponds to the minimal MRS value that allows EQ to stay above 0 along the optimal path with confidence level $\alpha=1 \%$. Parameter $\theta$ is determined from the IPCC Fifth Assessment Report which estimates that 100 years after a 100 Gt $\mathrm{CO}_{2}$ pulse in the preindustrial atmosphere, there would remain $40 \%$ of the $100 \mathrm{Gt}$ $\mathrm{CO}_{2}$ emitted, while after 1000 years $25 \%$ would remain. Accordingly, denoting by $\hat{\ell}$ the preindustrial level of $\mathrm{CO}_{2}$, after an initial period $\ell_{0}=\hat{\ell}+100$, we have $\ell_{100}=\hat{\ell}+40$ and $\ell_{1000}=\hat{\ell}+25$. Using (19) without industrial interferences and solving the recursion gives

$$
\ell_{t}=\theta^{t}(\hat{\ell}+100)+\left(1-\theta^{t}\right)\left[\ell_{M}-\hat{e} /(1-\theta)\right]
$$

Using the IPCC's estimates for $\ell_{100}$ and $\ell_{1000}$ we obtain

$$
\ell_{M}-\hat{\ell}-\frac{\hat{e}}{1-\theta}=\frac{40-100 \theta^{100}}{1-\theta^{100}}=\frac{25-100 \theta^{1000}}{1-\theta^{1000}} .
$$

The last equality can be expressed as $1-5 x+4 x^{10}=0$ where $x=\theta^{100}$ which gives $\theta \approx(1 / 5)^{1 / 100} \approx 0.984$ independently of the choice of $\hat{\ell}$ and $\ell_{M}{ }^{25}$ We deduce parameter $\hat{e}$ by assuming that EQ has reached its long term equilibrium $e_{N}=\ell_{M}-\hat{\ell}$ in the preindustrial period which gives $\hat{e}=(1-\theta) e_{N}$. Considering that $\hat{\ell}=2176 \mathrm{Gt} \mathrm{CO}_{2}$ $(280 \mathrm{ppm})$ and $\ell_{M}=5439 \mathrm{Gt} \mathrm{CO}_{2}(700 \mathrm{ppm})$, we obtain $\hat{e} \approx 52.1{ }^{26}$ Our reference year is 2005 which corresponds to a GHG level equal to $2945 \mathrm{Gt} \mathrm{CO}_{2}$ (379 ppm) hence an initial EQ index $e_{0}=2494.17 \mathrm{Gt} \mathrm{CO}_{2}$.

The AGT index in the reference year is deduced from the World Bank's estimates of the world $\mathrm{CO}_{2}$ intensity which in our framework is given by function 18 . ${ }^{27}$ The data show that the world $\mathrm{CO}_{2}$ intensity has sharply decreased over the second half of the 20th century and has been plateauing since 2000 (the period covered by the data is 1960 - 2013) with a value in the reference year $\iota_{2005}=0.51 \mathrm{~kg} / \mathrm{US} \$$. We assume

\footnotetext{
${ }^{24}$ The unit used in the following is the gigaton or Gt shorthand, i.e. $10^{9}$ metric tons. Theses levels are also commonly expressed in atmospheric concentration, the unit being the part per million or ppm shorthand, i.e. $0.01 \%$. Each ppm represents approximately $2.13 \mathrm{Gt}$ of carbon in the atmosphere as a whole, equivalent to $7.77 \mathrm{Gt}$ of $\mathrm{CO}_{2}$. Conversion values can be found on the dedicated US department of energy website http://cdiac.ornl.gov/pns/convert.html\#3.

${ }^{25}$ An obvious root of this equation is $x=1$. The other roots are complex numbers.

${ }^{26}$ According to the IPCC Fifth Assessment Report, $700 \mathrm{ppm}$ lead to a temperature increase of approximately $4^{\circ} \mathrm{C}$, a situation where "many global risks are high to very high." Acemoglu et al. (2012) use the atmospheric $\mathrm{CO}_{2}$ concentration that would lead to an approximate $6^{\circ} \mathrm{C}$ increase in temperatures. Stern (2007) reports that increases in temperature of more than $5^{\circ} \mathrm{C}$ will lead among other things to the melting of the Greenland Ice Sheet.

${ }^{27}$ The data can be found on the world bank website: http://data.worldbank.org/indicator/EN.ATM.CO2E.KD.GD;
} 
in our baseline setup (table 1) that the maximum emission intensity $\varphi$ is equal to $6.5 \mathrm{~kg} / \mathrm{US} \$$ (for static comparative exercises, we also consider the cases $\varphi=7.5 \mathrm{~kg} / \mathrm{US} \$$ and $\varphi=5 \mathrm{~kg} / \mathrm{US} \$$, see tables 6 and 7), so that the level of the AGT index in the reference year is given by $\mu_{2005}=q_{2005}\left(\varphi-\iota_{2005}\right) / \xi$ where $q_{2005}=47$ trillions US $\$$. The effectiveness of the green technologies is captured by the ratio $\xi / \varphi$. It is set to $1 / 2$ is the baseline setup, and we also consider the strong effectiveness $(\xi / \varphi=1)$ and the weak effectiveness $(\xi / \varphi=1 / 3)$ cases (cf. tables 4 and 5 ).

Parameters of the GDP dynamics (1) are set to $g=0.99$ and $\hat{g}=0.2$. These values correspond to a growth rate of $3.1 \%$ for the first year (2005) and a growth rate of $0.9 \%$ in year $2055(t=50)$ and then $0.4 \%$ in year $2105(t=100){ }^{28}$ Risk aversion coefficient $\gamma$ is set to 3 and $\psi$ at $10 \%$ which corresponds to a stationary state value of the interest rate $r_{S}$ of approximately $6 \%$ in the baseline scenario. The standard deviation of the shocks affecting the GDP is set to $\sigma_{\kappa}=1.5$ (trillions US $\$$ ), which corresponds to $3.19 \%$ of the 2005 GDP value. We also consider small and large GDP shocks, these cases corresponding to $\sigma_{\kappa}=2$ (table 2) and $\sigma_{\kappa}=1$ (table 3) respectively. The shocks affecting firms' beliefs are set to $\sigma_{\varepsilon}=\sigma_{\eta}=0.01$ (trillions US\$), hence $0.21 \%$ of the 2005 GDP value in the baseline. Table 8 reports values assuming $\sigma_{\eta}=1$ which is awfully large, but small variations around the baseline proved insignificant (we examined the cases where they are tenfold that of the baseline values and found no significant effects). For each set of parameters, the consumer's MRS $\rho$ is derived from (35) so that the interest rate matches its 2005 value, equal to $6 \%{ }^{29}$ All tables show the resulting estimates when $\lambda$ is ranging from 0 to 0.9 by decimal steps.

\section{Results}

[Table 1 about here.]

Table 1 presents the results of our baseline scenario. For each value of $\lambda$ indicated in the first column, the second column indicates the implied consumer's valuation of the environment $\rho$ given the other parameter values. The following columns $\sigma_{y}, \sigma_{\mu}$ and $r_{S}$ give the corresponding values of the (one period) standard deviation of the total wealth, $y_{t}=c_{t}+\rho e_{t}$, and of the AGT index $\mu_{t}$ respectively, and the longterm stationary level of the interest rate. The rest of the table reports the environmental policy parameters.

\footnotetext{
${ }^{28}$ In Acemoglu et al. (2012), the innovation function is calibrated so as to obtain a $2 \%$ long run growth. In DICE 2010, the economy grows at a rate equal to $1.9 \%$ from 2010 to 2100 and $0.9 \%$ from 2100 to 2200 .

${ }^{29}$ See King \& Low (2014), for a measure of world interest rates. According to this study, the 2005 quarterly values of the interest rate are $1.479 \%, 1.456 \%, 1.449 \%$ and $1.542 \%$.
} 
Column $\rho^{*}$ gives the CCVE at confidence level 1\%o, column $r_{\sharp}^{\star}$ the corresponding long term expected interest rate during the transitory phase toward an ENP, column $T$ indicates the date at which the expected EQ level under this policy merges with the expected ENP. A value equal to 199 indicates that the expected EQ does not reach the ENP in that time horizon (the maximum considered in the simulations). Finally, column $e_{T}$ indicates the expected EQ level reached a date $T$ under the policy.

We observe that standard deviations do not evolve monotonically with $\lambda: \sigma_{y}$ first decreases and then increases, while the reverse is true for $\sigma_{\mu}$. This is due to the changes in coefficients $\left\{a_{k}\right\}$ that are triggered by the variations of $\lambda$ and $\rho$. The stationary level of the interest rate $r_{S}$ is also non-monotonic: it first increases and then decreases. Still, it stays relatively close to $r_{0}=6 \%$. The expected optimal interest rate $r_{\sharp}^{\star}$ targeted by the social planner is particularly high compared to $r_{S}$ : while $r_{S}$ stays around $6 \%, r_{\sharp}^{\star}$ ranges from $18 \%$ to almost $33 \%$.

The consumer's valuation $\rho$ is very large for low values of $\lambda$ and decreases as $\lambda$ increases, while the trend for the CCVE $\rho^{\star}$ is the opposite: it increases with $\lambda$. One can observe that in this baseline case, $\rho^{*}$ is lower than $\rho$ as long as $\lambda$ is lower than 0.7. For example, if $\lambda=0.5$, the CCVE is $15.93 \$ / \mathrm{t}$, while the implicit consumers' valuation is $23.79 \$ / \mathrm{t}^{30}$ Graphic 3 depicts the evolutions of theses values and shows that they cross approximately around $\lambda=0.65$.

[Figure 3 about here.]

Finally, under the baseline scenario, carbon neutrality is a very long term objective: only when $\lambda$ is larger than 0.7 is carbon neutrality reached within two centuries (but less than a century if $\lambda$ is very large).

The fact that $\rho$ decreases with $\lambda$ is easily understood. The larger $\lambda$, the less dependent is the economy on past values of the AGT index and the more are investments reactive to market prices (which would graphically correspond to a flat capital demand curve). Under laissez-faire, when $\lambda$ is large consumers expect a low demand of AGT investments and thus an increase in consumption and a decrease in EQ over time. As we calibrate the model so that the interest rate matches its 2005 value, equal to $6 \%$, whatever the value of $\lambda$, it comes from (35) that $\rho$ should be the lower the larger $\lambda$. The impact of $\lambda$ on $r_{\#}^{\star}$ may come from two channels: a direct one, through the covariance term of (40) and an indirect one through changes in $\rho^{\star}$. Numerical computations of the

\footnotetext{
${ }^{30} \mathrm{We}$ can compare these values to the social costs of carbon found in the literature. Nordhaus (2007), finds a SCC of $11.5 \$ / \mathrm{t} \mathrm{CO}_{2}$ for 2015. More recently, with similar calibration for the rate of pure time preference, Traeger (2015) and Golosov et al. (2014), find SCC of respectively 15.5 and 16.4 $\$ / \mathrm{t} \mathrm{CO}_{2}$.
} 
first effect with our baseline calibration show a negative but very small impact. On the other hand, (31) and fig. 1 show that $r_{\#}^{e}$ (and thus $r_{\#}^{\star}$ ) increases with $\rho$. Intuitively, the social planner must implement a larger subsidy when EQ matters more from a social viewpoint, which increases capital demand and results in a larger long run optimal expected interest rate. Hence, the increase in $r_{\#}^{\star}$ with $\lambda$ showed in table 1 comes from the sharp increase in the MRS $\rho^{\star}$. Therefore, the major impact of technological spillovers on the CCVE comes from their impact on catastrophic risk: a large subsidy is required when $\lambda$ is large because the risk of hitting the environmental tipping point is large. Otherwise, assuming that the subsidy is small while $\lambda$ is large, EQ would reaches a lower minimum than the one prescribed by the environmental safety constraint. The environmental path is then more risky and requires a larger $\operatorname{MRS} \rho^{\star}$ to compensate. Tables 2 and 3 allow to assess the effects of the magnitude of shocks affecting the GDP.

[Table 2 about here.]

[Table 3 about here.]

Not surprisingly, the standard deviations of $\tilde{y}_{t}$ and $\tilde{\mu}_{t}$ are increasing with $\sigma_{\kappa}$, whereas the implied consumers' valuation $\rho$ and the long term interest rate $r_{S}$ are decreasing. The policy parameters are also negatively affected by a change in $\sigma_{\kappa}$, but to a lower extent: Large shocks slightly lower the optimal interest rate $r_{\sharp}^{\star}$ and the CCVE, while the ENP is reached faster (and conversely for small shocks). Large GDP shocks lower the market interest rates, by increasing the precautionary motive for savings. This compensates increased variances and explains a lower CCVE. Variations in the effectiveness of the green technologies, reported in tables 4 and 5, have a intuitive impact on the policy parameters: they are larger the less the green technologies are effective.

[Table 4 about here.]

[Table 5 about here.]

Similarly, the variations in the environmental damages due to industrial emissions reported in tables 6 and 7 show that the more they impact the environment, the larger the policy parameters are.

[Table 6 about here.]

[Table 7 about here.] 
Finally, table 8 shows the impact of a variation in the public signal.

[Table 8 about here.]

It should be noted that the change in $\sigma_{\eta}$ is 100 times larger than the baseline value. Smaller values (a tenfold increase of either the public or the private signal standard deviation) did not show any significant effect. Nevertheless, the standard deviations of $\tilde{y}_{t}$ and $\tilde{\mu}_{t}$ are not much affected. Interestingly, the range of $\rho^{*}$ is larger than in the baseline (its smaller value is reduced while its larger value is increased) while $r_{\sharp}^{\star}$ is reduced (by approximately $3 \mathrm{US} \$ / \mathrm{t}$ ). Using our baseline calibration and assuming $\lambda=.8$, fig. 4 and fig. 5 illustrate the dynamics of the AGT and EQ indexes respectively, starting from the first period of the implementation of the environmental policy (2006). In these figures, the expected paths and the upper and lower limits of a $95 \%$ confidence interval around these paths are depicted. Stochastically generated shocks allow us to illustrate the difference between a laissez-faire situation and the corresponding "realized" paths of the indexes under the environmental policy.

[Figure 4 about here.]

Also depicted fig. 4 is the carbon neutral path (the dashed blue curve indicated $\mu_{t}^{C N}$ ) that the expected value of the AGT index under the policy joins at date $T=147$. The laissez-faire curve shows a positive trend, but significantly below the one under the policy (actually, the curve stays below the lower-bound of the $95 \%$ interval). As a result, fig. 5 shows that EQ is rapidly deteriorating under laissez-faire (the carbon budget is entirely depleted in 25 years).

[Figure 5 about here.]

As mentioned above, EQ is also deteriorating under the optimal policy at first, reaching its minimum after approximately 80 years, but still sufficiently high to safely reduce the odds of reaching 0 . It then increases, but the level reached at the time the economy is carbon neutral $\left(e_{T}=1541 \mathrm{Gt}^{C_{2}}\right)$ is considerably lower than the one in $2005\left(e_{0}=2494 \mathrm{Gt}_{\mathrm{CO}_{2}}\right)$. The policy scheme $z_{t}$ and the resulting policy index $Z_{t}$ are illustrated fig. 6 .

[Figure 6 about here.] 
The subsidy $z_{t}$ increases rapidly the first 5 years (from $15 \%$ to $33 \%$ ) then slowly decreases toward $28 \%$ during the following century. As the targeted interest rate level is $27.58 \%$, the net expected subsidy rate is thus around $5 \%$. The actual level is stochastic as shown fig. 7 where the dynamics of the interest rate under the policy and laissezfaire are depicted together with the corresponding expected rates. Interest rates are similarly affected by the GDP shocks, the amplitude of the variations being larger under the policy than under laissez-faire.

[Figure 7 about here.]

Fig. 8 illustrates the per-period investment rates (relative to the AGT index level) under laissez-faire and under the environmental policy. The investment is negative under laissez-faire in the first 5 periods, that is, firms tend to invest increasingly in polluting technologies, while investment in green technology is always positive under the policy. Investment rates are comparable after 15 years (but not the level as noted above), and converge to the growth rate of the economy.

[Figure 8 about here.]

Fig. 9 shows the consumption path under the optimal policy and under laissez-faire. A higher consumption differential between laissez-faire and the regulated economy means that the policy implies a higher investment level and thus a higher cost in terms of postponed welfare.

[Figure 9 about here.]

Fig. 10 shows total wealth dynamics. Because of the decrease in the environmental quality, the laissez-faire curve decreases rapidly.

[Figure 10 about here.]

There are two aspects in the welfare impact of an environmental policy that can be analyzed here. First, implementing a subsidy for AGT has the immediate and most direct effect of increasing the rate of investment in green technologies, thus introducing a trade-off between current consumption and future environmental quality. Since our model disentangles green technology investment from productivity growth, increasing investment has no stock effect on the GDP level. On the other hand, the environmental quality is a stock and reducing the size of the negative impact of production on the environment has long term effects on welfare through environmental quality accumulation. 


\section{Conclusion}

In this paper, we analyze the AGT process when demand spillovers are a key determinant of technical efficiency and when the economy is subject to uncertainty. We consider the problem of a social planner in charge of determining an investment subsidy policy to incentivize firms to increase their AGT. Because investment choices are ultimately made by private agents who react to the economic context according to their beliefs and because the efficiency of technologies is the result of their many choices, the public policy can only imperfectly direct the economy to a sustainable path. Besides, the value that should be ascribed to the environment to guide the public policy is difficult to assess because of the lack of scientific knowledge and the uncertainty that affects the economy. While extremely stylized, our model allows us to ascertain the effects of these uncertainties on the optimal policy. It provides a tool to estimate the value of the environment considering that society should decide on a safety level in order to avoid an environmental catastrophe. Of course, the CCVE values that we obtain depend on the safety level chosen, but more importantly, they are very dependent of the spillover effects at work in the AGT process. Our numerical simulations show that the sharper the demand spillovers are, the higher is the CCVE and the larger should be the subsidies given to firms to direct their choices toward a carbon neutral path. We have considered in this analysis that the productivity growth is unaffected by the technologies employed: technologies only differ in their impact on the environment. Including in the analysis the process that leads to productivity growth would permit to assess the intertemporal trade-off between growth, consumption and environmental safety when society faces both economic and environmental risks. This undertaking could also includes other policy instruments than investment subsidies, like the environmental tax, and would certainly give better estimates of the CCVE. 


\section{References}

Acemoglu, D., Aghion, P., Bursztyn, L., \& Hemous, D. (2012). The environment and directed technical change. American Economic Review, 102(1), 131-66. 1, 26, 28

Angeletos, G.-M. \& Pavan, A. (2004). Transparency of information and coordination in economies with investment complementarities. The American economic review, 2 , 91-98. 10

Angeletos, G.-M. \& Pavan, A. (2007). Efficient use of information and social value of information. Econometrica, 4, 1103-1142. 10

Battisti, G. (2008). Innovations and the economics of new technology spreading within and across users: gaps and way forward. Journal of Cleaner Production, 16(1, Supplement 1), S22 - S31. Diffusion of cleaner technologies: Modeling, case studies and policy. 9

Bommier, A., Lanz, B., \& Zuber, S. (2015). Models-as-usual for unusual risks? on the value of catastrophic climate change. Journal of Environmental Economics and Management, 74, 1 - 22. 15

Bovenberg, A. L. \& Smulders, S. (1995). Environmental quality and pollutionaugmenting technological change in a two-sector endogenous growth model. Journal of Public Economics, 57, 369-391. 1

Bretschger, L. \& Vinogradova, A. (2014). Growth and Mitigation Policies with Uncertain Climate Damage. Working Paper 5085, CESifo. 6

Charnes, A. \& Cooper, W. W. (1959). Chance-constrained programming. Management Science, 6(1), 73-79. 14

De Zeeuw, A. \& Zemel, A. (2012). Regime shifts and uncertainty in pollution control. Journal of Economic Dynamics and Control, 36, 939-950. 6

Eaton, J. \& Kortum, S. (1999). International technology diffusion: Theory and measurement. International Economic Review, 40(3), 537-570. 1

Fischer, C. \& Heutel, G. (2013). Environmental macroeconomics: Environmental policy, business cycles, and directed technical change. Annu. Rev. Resour. Econ., 5(1), 197-210. 1

Gerlagh, R. \& Kuik, O. (2007). Carbon Leakage with International Technology Spillovers. Working Paper 2007.33, Fondazione Eni Enrico Mattei. 1

Golosov, M., Hassler, J., Krusell, P., \& Tsyvinski, A. (2014). Optimal taxes on fossil fuel in general equilibrium. Econometrica, 82(1), 41-88. 1, 30 
Heutel, G. (2012). How should environmental policy respond to business cycles? optimal policy under persistent productivity shocks. Review of Economic Dynamics, 15, 244-264. 1

Jensen, S. \& Traeger, C. P. (2014). Optimal climate change mitigation under longterm growth uncertainty: Stochastic integrated assessment and analytic findings. European Economic Review, 69, 104-125. 1

King, M. \& Low, D. (2014). Measuring the"world"real interest rate. Technical report, National Bureau of Economic Research. 29

Lucas, R. E. (2009). Ideas and growth. Economica, 76(301), 1-19. 1

Lucas Jr, R. E. \& Moll, B. (2014). Knowledge growth and the allocation of time. Journal of Political Economy, 122(1). 1

Morris, S. \& Shin, H. S. (2002). Social value of public information. The American economic review, 5, 1521-1534. 10

Morris, S. \& Shin, H. S. (2003). Global Games: Theory and Applications, volume 1 of Econometric Society Monographs, chapter 3, (pp. 56-114). Cambridge University Press. 3

Nordhaus, W. (2014). Estimates of the social cost of carbon: Concepts and results from the dice-2013r model and alternative approaches. Journal of the Association of Environmental and Resource Economists, 1(1/2), 273-312. 5

Nordhaus, W. D. (2007). A review of the stern review on the economics of climate change. Journal of economic literature, 45(3), 686-702. 30

Perla, J. \& Tonetti, C. (2014). Equilibrium imitation and growth. Journal of Political Economy, 122(1), 52-76. 1

Shapiro, A., Dentcheva, D., \& Ruszczy?ski, A. (2009). Lectures on Stochastic Programming: Modeling and Theory. MOS-SIAM Series on Optimization. Society for Industrial and Applied Mathematics. 3

Soretz, S. (2007). Efficient dynamic pollution taxation in an uncertain environment. Environmental and Resource Economics, 36, 57-84. 6

Stern, N. H. (2007). The economics of climate change: the Stern review. cambridge University press. 26

Stockey, N. L. (1998). Are there limitations to growth? International Economic Review, 39(1), 1-31. 1

Traeger, C. P. (2015). Analytic integrated assessment and uncertainty. Available at SSRN 2667972. 1, 30 
Tsur, Y. \& Zemel, A. (1998). Pollution control in an uncertain environment. Journal of Economic Dynamics and Control, 22, 967-975. 6

Tsur, Y. \& Zemel, A. (2008). Regulating environmental threats. Environmental and Resource Economics, 39(3), 297-310. 15

Tsur, Y. \& Zemel, A. (2009). Endogenous discounting and climate policy. Environmental and Resource Economics, 44(4), 507. 15

Weitzman, M. (2013). Tail-hedge discounting and the social cost of carbon. Journal of Economic Literature, 51(3), 873-882. 23

Yaari, M. E. (1965). Uncertain lifetime, life insurance, and the theory of the consumer. The Review of Economic Studies, 32(2), 137-150. 14 


\section{Appendix (For Online Publication)}

\section{A Proof of Proposition 1}

Minimizing (8) with respect to $I_{i t}$ leads to the first-order condition

$$
-1+\delta_{t} \mathbb{E}\left[\mathcal{V}\left(x_{i t}+I_{i t} ; \tilde{x}_{i t+1}^{\star}\right) / \partial x \mid \omega_{t}, w_{i t}\right]=0
$$

while the envelop condition yields

$$
\mathcal{V}\left(x_{i t} ; x_{i t}^{\star}\right) / \partial x=x_{i t}^{\star}-x_{i t}+\delta_{t} \mathbb{E}\left[\mathcal{V}\left(x_{i t}+I_{i t} ; \tilde{x}_{i t+1}^{\star}\right) / \partial x \mid \omega_{t}, w_{i t}\right]
$$

implying $\partial \mathcal{V}\left(x_{i t} ; x_{i t}^{\star}\right) / \partial x=x_{i t}^{\star}-x_{i t}+1$. Plugging this expression in (47) evaluated in expectation for period $t+1$ yields

$$
1+r_{t}=\mathbb{E}\left[x_{i t+1}^{\star}-\left(x_{i t}+I_{i t}\right)+1 \mid \omega_{t}, w_{i t}\right]=\hat{x}_{i t+1}-\left(x_{i t}+I_{i t}\right)+1
$$

Reorganizing terms gives (9). Replacing into (2) and using (6), we obtain that firm $i$ next period mix satisfies

$$
x_{i t+1}=x_{t+1}^{\star}+\tau \eta_{t}+(1-\tau) \varepsilon_{i t}-r_{t} .
$$

As $\int \varepsilon_{i t} d i=0$, we have

$$
\mu_{t+1}=\int_{i} x_{i t+1} d i=x_{t+1}^{\star}+\tau \eta_{t}-r_{t}
$$

and thus $x_{i t+1}=\mu_{t+1}+(1-\tau) \varepsilon_{i t}$. As idiosyncratic investments depend on the firms' current technologies and on signals that are normally distributed, their next period tech-

nologies are also normally distributed around $\mu_{t+1}$ with variance $\mathbb{V}\left[x_{i t+1}\right]=(1-\tau)^{2} \sigma_{\varepsilon}^{2}$. Using (49) to substitutes for $x_{t+1}^{\star}$ in (5) yields $\mu_{t+1}-\left(\tau \eta_{t}-r_{t}\right)=\mu_{t}+\lambda\left(\mu_{t+1}-\mu_{t}\right)$ which gives 10 .

\section{B Proof of Lemma 1}

At each date $t$, the Bellman equation corresponding to (14) can be written as

$$
v\left(S_{t-1} ; e_{t}\right)=\max _{s_{t}} u_{\phi}\left(r_{t-1} S_{t-1}+R_{t}-s_{t}, e_{t}\right)+\beta \mathbb{E}_{t} v\left(S_{t-1}+s_{t} ; \tilde{e}_{t+1}\right)
$$


where $S_{t}$ and $s_{t}$ are the state and the control variables respectively. The first-order equation is given by

$$
\partial u_{\phi}\left(c_{t}, e_{t}\right) / z_{t}-r_{t}+\tau \eta_{t}=\beta \mathbb{E}_{t}\left[\partial v\left(S_{t} ; \tilde{e}_{t+1}\right) / \partial S\right]
$$

and the envelope theorem gives

$$
\partial v\left(S_{t-1} ; e_{t}\right) / \partial S=r_{t-1} \partial u_{\phi}\left(c_{t}, e_{t}\right) / \partial c+\beta \mathbb{E}_{t}\left[\partial v\left(S_{t} ; \tilde{e}_{t+1}\right) / \partial S\right]
$$

Replacing the last term using (50), we get

$$
\partial v\left(S_{t-1} ; e_{t}\right) / \partial S=\left(1+r_{t-1}\right) \partial u_{\phi}\left(c_{t}, e_{t}\right) / \partial c
$$

Taking the expectation and replacing in (50) yields (15) where $1+r_{t}$ on the RHS is factorized out of the expected value since the date- $t$ interest rate is a known parameter.

\section{Proof of Proposition 2}

From (22) and (23), the first-order condition with respect to $z_{t}$ is given by

$$
\mathbb{E}\left[\partial u_{\phi t} / \partial c\right]=\beta \mathbb{E}\left[\partial \mathcal{W}_{t+1} / \partial \mu\right]
$$

where $u_{\phi t}$ and $\mathcal{W}_{t}$ are abbreviated notations for $u_{\phi}\left(c_{t}, e_{t}\right)$ and $\mathcal{W}\left(\mu_{t}, e_{t}, q_{t}\right)$ respectively. The envelop theorem gives

$$
\partial \mathcal{W}_{t} / \partial \mu=\xi \beta \mathbb{E}\left[\partial \mathcal{W}_{t+1} / \partial e\right]+\beta \mathbb{E}\left[\partial \mathcal{W}_{t+1} / \partial \mu\right]
$$

and

$$
\partial \mathcal{W}_{t} / \partial e=\mathbb{E}\left[\partial u_{\phi t} / \partial e\right]+\theta \beta \mathbb{E}\left[\partial \mathcal{W}_{t+1} / \partial e\right]
$$

Using (51), 52 can be written as

$$
\partial \mathcal{W}_{t} / \partial \mu=\xi \beta \mathbb{E}\left[\partial \mathcal{W}_{t+1} / \partial e\right]+\mathbb{E}\left[\partial u_{\phi t} / \partial e\right]
$$

Evaluating (54) in expectation one period ahead gives

$$
\mathbb{E}\left[\partial \mathcal{W}_{t+1} / \partial \mu\right]=\mathbb{E}\left[\partial u_{\phi t+1} / \partial c\right]+\xi \beta \mathbb{E}\left[\partial \mathcal{W}_{t+2} / \partial e\right]=\mathbb{E}\left[\partial u_{\phi t} / \partial c\right] / \beta
$$


using (51), hence

$$
\mathbb{E}\left[\partial \mathcal{W}_{t+2} / \partial e\right]=\mathbb{E}\left[\partial u_{\phi t} / \partial c\right] /\left(\xi \beta^{2}\right)-\mathbb{E}\left[\partial u_{\phi t+1} / \partial c\right] /(\xi \beta)
$$

Plugging this expression in (53) evaluated one period ahead yields

$$
\mathbb{E}\left[\partial \mathcal{W}_{t+1} / \partial e\right]=\mathbb{E}\left[\partial u_{\phi t+1} / \partial e\right]+\left(\mathbb{E}\left[\partial u_{\phi t} / \partial c\right] / \beta-\mathbb{E}\left[\partial u_{\phi t+1} / \partial c\right]\right) \theta / \xi
$$

We can thus express (54) as

$$
\frac{\partial \mathcal{W}_{t}}{\partial \mu}=(1+\theta) \mathbb{E}\left[\partial u_{\phi t} / \partial c\right]+\xi \beta \mathbb{E}\left[\partial u_{\phi t+1} / \partial e\right]-\theta \beta \mathbb{E}\left[\partial u_{\phi t+1} / \partial c\right]
$$

which, evaluated one period ahead yield gives,

$$
\mathbb{E}\left[\frac{\partial \mathcal{W}_{t+1}}{\partial \mu}\right]=(1+\theta) \mathbb{E}\left[\frac{\partial u_{\phi t+1}}{\partial c}\right]+\xi \beta \mathbb{E}\left[\frac{\partial u_{\phi t+2}}{\partial e}\right]-\theta \beta \mathbb{E}\left[\frac{\partial u_{\phi t+2}}{\partial c}\right]=\frac{1}{\beta} \mathbb{E}\left[\frac{\partial u_{\phi t}}{\partial c}\right]
$$

using (51). Reorganizing terms, we obtain

$$
\mathbb{E}\left[\frac{\partial u_{\phi t}}{\partial c}\right]=(1+\theta) \beta \mathbb{E}\left[\frac{\partial u_{\phi t+1}}{\partial c}\right]-\theta \beta^{2} \mathbb{E}\left[\frac{\partial u_{\phi t+2}}{\partial c}\right]+\beta^{2} \xi \mathbb{E}\left[\frac{\partial u_{\phi t+2}}{\partial e}\right] .
$$

From (24) and 27), we have

$$
\mathbb{E}\left[\partial u_{\phi t} / \partial c\right]=\left(1+r_{t}^{e}\right) \beta \mathbb{E}\left[\partial u_{\phi t+1} / \partial c\right]
$$

Substituting in (55) for initial dates $t$ and $t+1$ yields

$$
\frac{\beta^{2}}{\delta_{t}^{e} \delta_{t+1}^{e}} \mathbb{E}\left[\frac{\partial u_{\phi t+2}}{\partial c}\right]=\frac{(1+\theta) \beta^{2}}{\delta_{t+1}^{e}} \mathbb{E}\left[\frac{\partial u_{\phi t+2}}{\partial c}\right]-\theta \beta^{2} \mathbb{E}\left[\frac{\partial u_{\phi t+2}}{\partial c}\right]+\beta^{2} \xi \mathbb{E}\left[\frac{\partial u_{\phi t+2}}{\partial e}\right]
$$

where $\delta_{t}^{e} \equiv\left(1+r_{t}^{e}\right)^{-1}$, which upon simplifying and rearranging terms yields

$$
\begin{aligned}
\xi \mathbb{E}\left[\partial u_{\phi t+2} / \partial e\right] / \mathbb{E}\left[\partial u_{\phi t+2} / \partial c\right] & =\left(1+r_{t}^{e}\right)\left(1+r_{t+1}^{e}\right)-\left(1+r_{t}^{e}\right)(1+\theta)+\theta \\
& =r_{t+1}^{e}+r_{t}^{e} r_{t+1}^{e}-r_{t}^{e} \theta
\end{aligned}
$$




\section{Proof of Proposition 3}

Expression (28) gives the recursive equation

$$
r_{t+1}^{e}=\left(\rho \xi+r_{t}^{e} \theta\right) /\left(1+r_{t}^{e}\right)
$$

which can be solved as follows. Defining $v_{t}=\left(r_{t}^{e}+c\right)^{-1}$ or equivalently $r_{t}^{e}=1 / v_{t}-c$ where $c$ is a constant to determine, (56) becomes

$$
\frac{1}{v_{t+1}}=\frac{v_{t}[c(1-c)+\rho \xi-\theta c]+c+\theta}{v_{t}(1-c)+1}
$$

which gives

$$
v_{t+1}=\frac{v_{t}(1-c)+1}{v_{t}[c(1-c-\theta)+\rho \xi]+c+\theta},
$$

an equation that simplifies to

$$
v_{t+1}=v_{t}(1-c) /(c+\theta)+(c+\theta)^{-1} \equiv v_{t} k+k_{0},
$$

under the conditions $c \neq-\theta$ and

$$
c(1-c-\theta)+\rho \xi=0 .
$$

Provided that $k \neq 1$, the solution of the recurrence equation (57) is given by

$$
v_{t+1}=k^{t+1} v_{0}+k_{0}\left(1-k^{t+1}\right) /(1-k)
$$

which converges to $v_{\infty}=k_{0} /(1-k)$ if $|k|<1$, i.e. if $1>|(1-c) /(c+\theta)|>0$, albeit with oscillations along its path if $k<0$. The corresponding solution of (28) would converge to

$$
r_{\infty}^{e}=(1-k) / k_{0}-c=\theta-1+c
$$

which must be equal to the solution of 29, i.e. we must have

$$
r_{\sharp}^{e}=\theta-1+c
$$

where $r_{\sharp}^{e}$ is either equal to $(A-1+\theta) / 2>0$ or $-(A+1-\theta) / 2<0$. Using (60) to substitute $r_{\sharp}^{e}+1-\theta$ for $c$ in (58) yields (29). (58) is thus satisfied if (60) is true. Using 
(60) and (57) yields

$$
k=\left(\theta-r_{\sharp}^{e}\right) /\left(r_{\sharp}^{e}+1\right) .
$$

We have $k>0$ if $-1<r_{\sharp}^{e}<\theta$ (as we cannot have $r_{\sharp}^{e}>\theta$ and $r_{\sharp}^{e}<-1$ ). The condition $k<1$ implies $\theta-r_{\sharp}^{e}<1+r_{\sharp}^{e}$, hence $(\theta-1) / 2<r_{\sharp}^{e}$. We thus have $1>k>0$ if $(\theta-1) / 2<r_{\sharp}^{e}<\theta$, which rules out the negative root of $(29)$, since we cannot have $(\theta-1) / 2<-(A+1-\theta) / 2$. The lower-bound condition on $r_{\sharp}^{e}$ gives $(\theta-1) / 2<$ $(A-1+\theta) / 2$, which is always true. The upper-bound condition on $r_{\sharp}^{e}$ can be written as $\theta>(A-1+\theta) / 2$ which gives $A<\theta+1$. Squaring both terms, we arrive at $4 \rho \xi<(1+\theta)^{2}-(1-\theta)^{2}=4 \theta$ hence $\rho \xi<\theta$.

We have $k<0$ if either $r_{\sharp}^{e}<-1$ or if $r_{\sharp}^{e}>\theta$. Moreover we have $|k|<1$ if $r_{\sharp}^{e}-\theta<1+r_{\sharp}^{e}$ which is always true. Hence, we have $-1<k<0$ if $r_{\sharp}^{e}>\theta$ or if $r_{\sharp}^{e}<-1$. Taking the positive root, this condition gives $A-1+\theta>2 \theta$ i.e. $A>1+\theta$ hence $\rho \xi>\theta$.

Substituting $(A-1+\theta) / 2$ for $r_{\sharp}^{e}$ in 61 yields 32 . From 60 , the condition $c \neq \theta$ is equivalently stated as $r_{\sharp}^{e} \neq-1$ which is always the case with the positive root. Using (59) and as initial value $r_{t_{0}}^{e}=1 / v_{t_{0}}-c$, the solution of (28) at period $t \geq t_{0}$ satisfies

$$
r_{t}^{e}=\frac{(1-k)\left(r_{t_{0}}^{e}+c\right)}{(1-k) k^{t-t_{0}}+k_{0}\left(1-k^{t-t_{0}}\right)\left(r_{t_{0}}^{e}+c\right)}-c .
$$

Using $(1-k) / k_{0}=r_{\sharp}^{e}+c$, we get

$$
r_{t}^{e}=r_{\sharp}^{e}+\frac{\left(r_{\sharp}^{e}+c\right)\left(r_{t_{0}}^{e}-r_{\sharp}^{e}\right) k^{t-t_{0}}}{r_{\sharp}^{e}+c+\left(1-k^{t-t_{0}}\right)\left(r_{t_{0}}^{e}-r_{\sharp}^{e}\right)} .
$$

Using (60) and (31), we obtain $r_{\sharp}^{e}+c=2 r_{\sharp}^{e}+1-\theta=A$. Substituting allows us to obtain 30 which converges to $r_{\sharp}^{e}$ when $r_{\sharp}^{e}>r_{t_{0}}^{e}$ if $A>r_{\sharp}^{e}-r_{t_{0}}^{e}$ hence $r_{t_{0}}^{e}>-(A+1-\theta) / 2$.

\section{E Proof of Proposition 4}

This result can be obtained recursively by observing that 30 can be rewritten in term of normalized gaps as $d_{t_{0}+h}=f^{h}\left(d_{t_{0}}\right)$ with $f^{0}(x)=x$ and $f^{h}(x) \equiv f \circ f^{h-1}(x)$ for all $h \geq 1$. Indeed, it is true for $h=1$ since $d_{t_{0}+1}=f\left(d_{t_{0}}\right)$ and supposing it is true for $t+h$, it is true for $t+h+1$ : we have $f\left(d_{t+h}\right)=f\left(f^{h}\left(d_{t}\right)\right)=f^{h+1}\left(d_{t}\right)=d_{t+h+1}$. A 
direct and alternative proof is obtained using $f^{h}(x)=k^{h} x /\left[1+\left(1-k^{h}\right) x\right]$ : we get $f\left(f^{h}(x)\right)=\frac{k f^{h}(x),}{1+(1-k) f^{h}(x)}=\frac{k^{h+1} x}{1+\left(1-k^{h}\right) x+(1-k) k^{h} x}=\frac{k^{h+1} x}{1+\left(1-k^{h+1}\right) x}=f^{h+1}(x)$.

\section{F Proof of Proposition5}

Using (1), (16) and (19), we have

$$
\tilde{y}_{t+1}=\tilde{c}_{t+1}+\rho e_{t+1}=g q_{t}+\hat{g}+\tilde{\kappa}_{t+1}-\left(\tilde{\mu}_{t+2}-\mu_{t+1}\right)+\rho\left(\theta e_{t}+\xi \mu_{t}-\varphi q_{t}+\hat{e}\right)
$$

in which, using (36),

$$
\tilde{\mu}_{t+2}=a_{1} \mu_{t+1}+a_{2}\left(\theta e_{t}+\xi \mu_{t}-\varphi q_{t}+\hat{e}\right)+a_{3}\left(g q_{t}+\hat{g}+\tilde{\kappa}_{t+1}\right)+a_{4}+a_{5}\left(\tau \tilde{\eta}_{t+1}\right)+Z_{t+1}
$$

where $Z_{t+1}$ is a function of the $z_{t+h}, h=2,3 \ldots$ As the resulting expression of $\tilde{y}_{t+1}$ is a linear combination of iid random shocks normally distributed, it is also normally distributed with variance $\sigma_{y}$ given by $(38)$ which is independent of $t$.

The coefficients $\left\{a_{j}\right\}_{j=1, \ldots, 6}$ and $Z_{t}$ in $(36)$ are derived as follows. Using $y_{t}=q_{t}-$ $\mu_{t+1}+\mu_{t}+\rho e_{t}$ yields

$$
\mathbb{E}_{t}\left[\tilde{y}_{t+1}\right]-y_{t}=\hat{g}-(1-g+\rho \varphi) q_{t}-\mathbb{E}_{t}\left[\tilde{\mu}_{t+2}\right]+2 \mu_{t+1}-(1-\rho \xi) \mu_{t}-\rho\left[(1-\theta) e_{t}-\hat{e}\right]
$$

which gives, using (63) and collecting terms,

$$
\begin{aligned}
\mathbb{E}_{t}\left[\tilde{y}_{t+1}\right]-y_{t} & =\hat{g}\left(1-a_{3}\right)-\left[1-\left(1-a_{3}\right) g+\left(\rho-a_{2}\right) \varphi\right] q_{t}-\left(a_{4}+Z_{t+1}\right)+\left(2-a_{1}\right) \mu_{t+1} \\
& -\left[1-\left(\rho-a_{2}\right) \xi\right] \mu_{t}-\left[\rho(1-\theta)+a_{2} \theta\right] e_{t}+\left(\rho-a_{2}\right) \hat{e}
\end{aligned}
$$

Replacing into 35 gives

$$
\begin{aligned}
r_{t} & =\psi-\gamma^{2} \sigma_{y}^{2} / 2+\gamma\left\{\left[\varphi a_{2}-(1-g+\varphi \rho)-a_{3} g\right] q_{t}-\left(a_{1}-2\right) \mu_{t+1}-\left[1+\xi\left(a_{2}-\rho\right)\right] \mu_{t}\right\} \\
& +\gamma\left\{\hat{g}\left(1-a_{3}\right)-e_{t}\left[a_{2} \theta+\rho(1-\theta)\right]-\hat{e}\left(a_{2}-\rho\right)-a_{4}-Z_{t+1}\right\} .
\end{aligned}
$$

Equalizing with (13), which can be rewritten as

$$
r_{t}=z_{t}+\tau \eta_{t}-(1-\lambda)\left(\mu_{t+1}-\mu_{t}\right),
$$


and collecting terms yields

$$
\begin{aligned}
\mu_{t+1} & =\frac{1-\lambda+\gamma\left[1+\xi\left(a_{2}-\rho\right)\right]}{1-\lambda+\gamma\left(2-a_{1}\right)} \mu_{t}+\gamma \frac{\left[a_{2} \theta+\rho(1-\theta)\right] e_{t}}{1-\lambda+\gamma\left(2-a_{1}\right)}+\gamma \frac{\left[1-g+\varphi\left(\rho-a_{2}\right)+a_{3} g\right] q_{t}}{1-\lambda+\gamma\left(2-a_{1}\right)} \\
& -\frac{\psi+\gamma \hat{g}\left(1-a_{3}\right)-\gamma \hat{e}\left(a_{2}-\rho\right)-\gamma a_{4}-\gamma^{2} \sigma_{y}^{2} / 2}{1-\lambda+\gamma\left(2-a_{1}\right)}+\frac{\tau \eta_{t}}{1-\lambda+\gamma\left(2-a_{1}\right)} \\
& +\frac{z_{t}+\gamma Z_{t+1}}{1-\lambda+\gamma\left(2-a_{1}\right)} .
\end{aligned}
$$

Identifying with 36 gives

$$
\begin{aligned}
& a_{1}=\frac{1-\lambda+\gamma\left[1+\xi\left(a_{2}-\rho\right)\right]}{1-\lambda+\gamma\left(2-a_{1}\right)}, a_{2}=\frac{\gamma \rho(1-\theta)}{1-\lambda+\gamma\left(2-a_{1}-\theta\right)}, a_{3}=\gamma \frac{1-g+\varphi\left(\rho-a_{2}\right)}{1-\lambda+\gamma\left(2-a_{1}-g\right)} \\
& a_{4}=\frac{\gamma\left[\hat{e}\left(a_{2}-\rho\right)+a_{4}+\gamma \sigma_{y}^{2} / 2\right]-\psi-\gamma \hat{g}\left(1-a_{3}\right)}{1-\lambda+\gamma\left(2-a_{1}\right)}, a_{5}=\frac{1}{1-\lambda+\gamma\left(2-a_{1}\right)} .
\end{aligned}
$$

and

$$
Z_{t}=a_{5}\left(z_{t}+\gamma Z_{t+1}\right)=a_{5} \sum_{i=0}^{+\infty}\left(a_{5} \gamma\right)^{i} z_{t+i} .
$$

The first two equations of (66) form a system involving only coefficients $a_{1}$ and $a_{2}$ that can be solved separately from the others. Observe also that $a_{2}=\rho$ and $a_{1}=a_{1}^{0} \equiv 1+(1-\lambda) / \gamma$ is a degenerate solution of this system. More precisely, using $1-\lambda+\gamma\left(2-a_{1}\right)=\gamma\left(a_{1}^{0}-a_{1}+1\right)$, we can express (66) as

$$
\begin{aligned}
& a_{1}=\frac{a_{1}^{0}+\xi\left(a_{2}-\rho\right)}{a_{1}^{0}-a_{1}+1}, a_{2}=\frac{\rho(1-\theta)}{a_{1}^{0}-a_{1}+1-\theta},, a_{3}=\frac{1-g+\varphi\left(\rho-a_{2}\right)}{a_{1}^{0}-a_{1}+1-g}, \\
& a_{5}=\frac{1}{\gamma\left(a_{1}^{0}-a_{1}+1\right)}, a_{4}=-\frac{\hat{e}\left(\rho-a_{2}\right)+\hat{g}\left(1-a_{3}\right)+\psi / \gamma-\gamma \sigma_{y}^{2} / 2}{a_{1}^{0}-a_{1}}
\end{aligned}
$$

and we get, assuming that the solution is $a_{2}=\rho$ and $a_{1}=a_{1}^{0}$, that $a_{3}=1, a_{5}=1 / \gamma$ but $a_{4}$ diverges unless $\psi=\gamma^{2} \sigma_{y}^{2} / 2=\tau^{2} \sigma_{\eta}^{2} / 2$ in which case $a_{4}$ is indefinite. Alternative solutions can be derived as follows. From the expression of $a_{2}$, we get

$$
a_{2}-\rho=\frac{-\rho\left(a_{1}^{0}-a_{1}\right)}{a_{1}^{0}-a_{1}+1-\theta}
$$

which, plugged into the expression for $a_{1}$, gives

$$
a_{1}\left(a_{1}^{0}-a_{1}+1\right)-a_{1}^{0}=\frac{-\rho \xi\left(a_{1}^{0}-a_{1}\right)}{a_{1}^{0}-a_{1}+1-\theta},
$$


that can also be expressed as $\left(a_{1}^{0}-a_{1}\right) P\left(a_{1}-1\right)=0$ where $P(x) \equiv x\left(a_{1}^{0}-\theta-x\right)+\rho \xi$ is a second degree polynomial. Non-degenerate solutions must solve $P\left(a_{1}-1\right)=0$. As $P(0)=P\left(a_{1}^{0}-\theta\right)=\rho \xi$ and $P(x)$ is concave, $a_{1}$ is either lower than $1\left(a_{1}-1\right.$ is equal to the negative root of $P(x)=0)$ or greater than $a_{1}^{0}-\theta+1=2-\theta+(1-\lambda) / \gamma\left(a_{1}-1\right.$ is then equal to the positive root of $P(x)=0)$. As $z_{t}$ impacts positively $\mu_{t+1}$ for all $t$, it comes from (67) that $a_{5}>0$ which implies $a_{1}-1<1+(1-\lambda) / \gamma$ and thus rules out the positive root of $P\left(a_{1}-1\right)=0$. Consequently, $a_{1}-1$ corresponds to the negative solution of $P(x)=0$ which is given by

$$
x=\frac{1}{2}\left(a_{1}^{0}-\theta-\sqrt{\left(a_{1}^{0}-\theta\right)^{2}+4 \rho \xi}\right) .
$$

After substituting $1+(1-\lambda) / \gamma$ for $a_{1}^{0}$, we get

$$
a_{1}=1-\frac{1}{2}\left(\sqrt{[1-\theta+(1-\lambda) / \gamma]^{2}+4 \rho \xi}-1+\theta-(1-\lambda) / \gamma\right),
$$

The condition $a_{1}>0$ can be expressed as

$$
[3-\theta+(1-\lambda) / \gamma]^{2}-[1-\theta+(1-\lambda) / \gamma]^{2}-4 \rho \xi>0
$$

which simplifies to $2+(1-\lambda) / \gamma-\theta>\rho \xi$. Using $a_{1}^{0}-\theta-a_{1}+1=\rho \xi /\left(1-a_{1}\right)$ in 68 gives

$$
a_{2}=\rho(1-\theta)\left(1-a_{1}\right) / \xi .
$$

and the other coefficients are deduced from

$a_{3}=\frac{\gamma\left[1-g+\varphi\left(\rho-a_{2}\right)\right]}{1-\lambda+\gamma\left(2-a_{1}-g\right)}, a_{4}=-\frac{\gamma\left[\hat{e}\left(\rho-a_{2}\right)+\hat{g}\left(1-a_{3}\right)\right]+r_{S}}{1-\lambda+\gamma\left(1-a_{1}\right)}, a_{5}=\frac{1}{1-\lambda+\gamma\left(2-a_{1}\right)}$.

As $a_{1}^{0}>1>a_{1}$ we have $a_{2}>0$ and from (69), $a_{2}<\rho$. We obtain from (68) that $a_{3}>0$, and we have $a_{3}<1$ if

$$
a_{1}^{0}-a_{1}>\varphi\left(\rho-a_{2}\right)=\frac{\varphi \rho\left(a_{1}^{0}-a_{1}\right)}{a_{1}^{0}-a_{1}+1-\theta}
$$

hence if $a_{1}^{0}-a_{1}+1-\theta>\varphi \rho$. From $P\left(a_{1}-1\right)=0$, we have $a_{1}^{0}-\theta-\left(a_{1}-1\right)=\rho \xi /\left(1-a_{1}\right)$. Replacing, the condition can be expressed as

$$
\xi / \varphi>1-a_{1}=\frac{1}{2}\left(\sqrt{[1-\theta+(1-\lambda) / \gamma]^{2}+4 \rho \xi}-1+\theta-(1-\lambda) / \gamma\right) .
$$


As the RHS of this inequality is increasing in $\rho$ and null when $\rho=0$, this is the case when $\rho$ is not too large. Since we assume $2+(1-\lambda) / \gamma-\theta>\rho \xi$ (to have $a_{1}>0$ ), a sufficient condition for $a_{3}<1$ is given by

$$
\frac{1}{2}\left(\sqrt{[1-\theta+(1-\lambda) / \gamma]^{2}+4[2+(1-\lambda) / \gamma-\theta]}-1+\theta-(1-\lambda) / \gamma\right) \leq \xi / \varphi
$$

which can be written as

$$
\frac{1}{2}\left(\sqrt{y^{2}+4 y+4}-y\right) \leq K
$$

where $y=1-\theta+(1-\lambda) / \gamma$ and $K=\xi / \varphi$, which gives

$$
0 \geq y^{2}+4 y+4-(2 K+y)^{2}=4 y(1-K)+4\left(1-K^{2}\right)=4(1-K)(y+1+K) .
$$

Consequently, a sufficient condition for $a_{3}<1$ is $\xi \geq \varphi$. We also have $a_{5}>0$ and $\gamma a_{5}<1$ if $\gamma<1-\lambda+\gamma\left(2-a_{1}\right)$ hence if $0<1-\lambda+\gamma\left(1-a_{1}\right)$ which is always the case since $a_{1}<1$. $Z_{t}$ is thus an exponential smoothing of the public policy scheme $\left\{z_{t+h}\right\}_{h \geq 0}$.

Differentiating $P\left(a_{1}-1\right)=0$ wrt $\lambda$ yields

$$
\frac{d a_{1}}{d \lambda}=\frac{-\left(1-a_{1}\right)}{\gamma\left[a_{1}^{0}-\theta+2\left(1-a_{1}\right)\right]}<0
$$

which also gives, using (71),

$$
\frac{d a_{2}}{d \lambda}=-\frac{\rho(1-\theta)}{\xi} \frac{d a_{1}}{d \lambda}>0
$$

Differentiating $a_{5}$ wrt $\lambda$ gives

$$
\frac{d a_{5}}{d \lambda}=a_{5}^{2}\left(1+\gamma \frac{d a_{1}}{d \lambda}\right)
$$

where, using $P\left(a_{1}-1\right)=0$,

$$
1+\gamma \frac{d a_{1}}{d \lambda}=\frac{a_{1}^{0}-\theta-\left(1-a_{1}\right)}{a_{1}^{0}-\theta+2\left(1-a_{1}\right)}=\frac{\rho \xi}{\left(1-a_{1}\right)\left[a_{1}^{0}-\theta+2\left(1-a_{1}\right)\right]}=-\frac{d a_{1}}{d \lambda} \frac{\gamma \rho \xi}{\left(1-a_{1}\right)^{2}}>0
$$

We thus have $d a_{5} / d \lambda>0$. Differentiating $a_{3}$ wrt $\lambda$ yields

$$
\frac{d a_{3}}{d \lambda}=\gamma \frac{-\varphi\left[1-\lambda+\gamma\left(2-a_{1}-g\right)\right]\left(d a_{2} / d \lambda\right)+\left[1-g+\varphi\left(\rho-a_{2}\right)\right]\left(1+\gamma d a_{1} / d \lambda\right)}{\left[1-\lambda+\gamma\left(2-a_{1}-g\right)\right]^{2}}
$$


where, using $a_{1}^{0}=1+(1-\lambda) / \gamma$,

$$
1-\lambda+\gamma\left(2-a_{1}-g\right)=\gamma\left(a_{1}^{0}-a_{1}+1-g\right)
$$

and, using (69) and (71),

$$
\rho-a_{2}=\frac{\rho\left(a_{1}^{0}-a_{1}\right)}{a_{1}^{0}-a_{1}+1-\theta}=\frac{\left(a_{1}^{0}-a_{1}\right)\left(1-a_{1}\right)}{\xi} .
$$

Replacing, we obtain that $d a_{3} / d \lambda>0$ if

$$
\begin{aligned}
0 & >\frac{\varphi(1-\theta)}{\xi}\left(a_{1}^{0}-a_{1}+1-g\right)-\frac{\xi}{\left(1-a_{1}\right)^{2}}\left[1-g+\varphi \frac{\left(a_{1}^{0}-a_{1}\right)\left(1-a_{1}\right)}{\xi}\right] \\
& =(1-g)\left(\frac{\varphi(1-\theta)}{\xi}-\frac{\xi}{\left(1-a_{1}\right)^{2}}\right)+\varphi\left(a_{1}^{0}-a_{1}\right)\left[\frac{1-\theta}{\xi}-\frac{1}{1-a_{1}}\right] \\
& =\frac{(1-g) \xi}{\left(1-a_{1}\right)^{2}}\left(\frac{\varphi a_{2}^{2}}{(1-\theta) \rho^{2}}-1\right)+\frac{\varphi\left(a_{1}^{0}-a_{1}\right)}{1-a_{1}}\left(\frac{a_{2}}{\rho}-1\right) .
\end{aligned}
$$

As $a_{2}<\rho$, this is always the case if $a_{2}<\rho \sqrt{(1-\theta) / \varphi}$, hence if $a_{1}>1-$ $\xi / \sqrt{(1-\theta) \varphi}$ which yields

$$
\frac{1}{2}\left(\sqrt{[1-\theta+(1-\lambda) / \gamma]^{2}+4 \rho \xi}-1+\theta-(1-\lambda) / \gamma\right)<\xi / \sqrt{(1-\theta) \varphi} .
$$

As the LHS of this inequality is increasing in $\rho$ and null when $\rho=0$, we thus have $d a_{3} / d \lambda>0$ when $\rho$ is not too large. Since we assume $2+(1-\lambda) / \gamma-\theta>\rho \xi$ (to have $\left.a_{1}>0\right)$, a sufficient condition for $d a_{3} / d \lambda>0$ is given by

$\frac{1}{2}\left(\sqrt{[1-\theta+(1-\lambda) / \gamma]^{2}+4[2+(1-\lambda) / \gamma-\theta]}-1+\theta-(1-\lambda) / \gamma\right)<\xi / \sqrt{(1-\theta) \varphi}$

which can be written as

$$
\frac{1}{2}\left(\sqrt{y^{2}+4 y+4}-y\right)<K
$$

where $y=1-\theta+(1-\lambda) / \gamma$ and $K=\xi / \sqrt{(1-\theta) \varphi}$, which gives

$$
0>y^{2}+4 y+4-(2 K+y)^{2}=4 y(1-K)+4\left(1-K^{2}\right)=4(1-K)(y+1+K) .
$$

Consequently, a sufficient condition for $a_{3}$ to increase with $\lambda$ is $\xi>\sqrt{(1-\theta) \varphi}$. 


\section{G Proof of lemma 2}

Using (64), we get

$$
\tilde{r}_{t}-\mathbb{E}_{t-1}\left[\tilde{r}_{t}\right]=\gamma\left\{\left[\varphi a_{2}-(1-g+\varphi \rho)-g a_{3}-\left(a_{1}-2\right) a_{3}\right] \tilde{\kappa}_{t}-\left(a_{1}-2\right) a_{5}\left(\tau \tilde{\eta}_{t}\right)\right\} .
$$

Using the expressions of $a_{3}$ and $a_{5}$ in (66), it can be expressed as

$$
\tilde{r}_{t}-\mathbb{E}_{t-1}\left[\tilde{r}_{t}\right]=\left[1-(1-\lambda) a_{5}\right]\left(\tau \tilde{\eta}_{t}\right)-(1-\lambda) a_{3} \tilde{\kappa}_{t}
$$

that can also be derived from (65), which becomes

$$
\tilde{r}_{t}=z_{t}+\tau \tilde{\eta}_{t}-(1-\lambda)\left(\tilde{\mu}_{t+1}-\mu_{t}\right)
$$

using (36). The two-period-ahead wealth index is deduced from

$$
\tilde{y}_{t+1}=\tilde{c}_{t+1}+\rho \tilde{e}_{t+1}=\tilde{q}_{t+1}+\tilde{\mu}_{t+1}-\tilde{\mu}_{t+2}+\rho \tilde{e}_{t+1}
$$

where

$$
\tilde{\mu}_{t+2}=a_{1} \tilde{\mu}_{t+1}+a_{2} \tilde{e}_{t+1}+a_{3} \tilde{q}_{t+1}+a_{4}+a_{5}\left(\tau \tilde{\eta}_{t+1}\right)+Z_{t+1} .
$$

Replacing leads to

$$
\tilde{y}_{t+1}=\left(1-a_{3}\right) \tilde{q}_{t+1}+\left(1-a_{1}\right) \tilde{\mu}_{t+1}+\left(\rho-a_{2}\right) \tilde{e}_{t+1}-a_{4}-a_{5}\left(\tau \tilde{\eta}_{t+1}\right)-Z_{t+1}
$$

which gives

$$
\begin{aligned}
\tilde{y}_{t+1}-\mathbb{E}\left[\tilde{y}_{t+1}\right] & =\left(1-a_{3}\right)\left(g \tilde{\kappa}_{t}+\tilde{\kappa}_{t+1}\right)+\left(1-a_{1}\right)\left[a_{3} \tilde{\kappa}_{t}+a_{5}\left(\tau \tilde{\eta}_{t}\right)\right]+\left(a_{2}-\rho\right) \varphi \tilde{\kappa}_{t}-a_{5}\left(\tau \tilde{\eta}_{t+1}\right) \\
& =\left(1-a_{3}\right) \tilde{\kappa}_{t+1}-a_{5}\left(\tau \tilde{\eta}_{t+1}\right)+\left[\left(1-a_{3}\right) g+\left(1-a_{1}\right) a_{3}+\left(a_{2}-\rho\right) \varphi\right] \tilde{\kappa}_{t}+a_{5}\left(1-a_{1}\right)\left(\tau \tilde{\eta}_{t}\right)
\end{aligned}
$$

and

$$
\begin{aligned}
\mathbb{V}\left[\tilde{y}_{t+1}\right] & =\left(1-a_{3}\right)^{2} \sigma_{\kappa}^{2}+a_{5}^{2} \tau^{2} \sigma_{\eta}^{2}+\left[\left(1-a_{3}\right) g+\left(1-a_{1}\right) a_{3}+\left(a_{2}-\rho\right) \varphi\right]^{2} \sigma_{\kappa}^{2} \\
& +\left(1-a_{1}\right)^{2} a_{5}^{2} \tau^{2} \sigma_{\eta}^{2} \\
& =\sigma_{y}^{2}+\left[\left(1-a_{3}\right) g+\left(1-a_{1}\right) a_{3}+\left(a_{2}-\rho\right) \varphi\right]^{2} \sigma_{\kappa}^{2}+\left(1-a_{1}\right)^{2} a_{5}^{2} \tau^{2} \sigma_{\eta}^{2} \equiv \sigma_{y_{+1}}^{2}
\end{aligned}
$$




\section{H Proof of Proposition 6}

The expected AGT index satisfies $\mathbb{E}\left[\tilde{\mu}_{t}\right]=\mathbb{E}\left[\tilde{q}_{t}\right] \varphi / \xi$ for all $t>T$ along an ENP, so that expected wealth satisfies

$$
\begin{aligned}
\mathbb{E}\left[\tilde{y}_{t}\right] & =\mathbb{E}\left[\tilde{c}_{t}\right]+\rho \mathbb{E}\left[\tilde{e}_{t}\right]=\mathbb{E}\left[\tilde{q}_{t}\right]-\mathbb{E}\left[\tilde{\mu}_{t+1}\right]+\mathbb{E}\left[\tilde{\mu}_{t}\right]+\rho \mathbb{E}\left[\tilde{e}_{t}\right] \\
& =\mathbb{E}\left[\tilde{q}_{t}\right][1+(1-g) \varphi / \xi]-\hat{g} \varphi / \xi+\rho \mathbb{E}\left[\tilde{e}_{t}\right] .
\end{aligned}
$$

while it is given by

$$
\mathbb{E}\left[\tilde{y}_{t}\right]=\mathbb{E}\left[\tilde{q}_{t}\right][1+(1-g) \varphi / \xi]-\hat{g} \varphi / \xi+\rho e_{T}
$$

along an SEP. From (35), the expected interest rate along an ENP satisfies

$$
\begin{aligned}
\mathbb{E}\left[\tilde{r}_{t}\right] & =r_{S}+\gamma\left(\mathbb{E}\left[y_{t+1}\right]-\mathbb{E}\left[y_{t}\right]\right) \\
& =r_{S}+\gamma\left(\mathbb{E}\left[\tilde{q}_{t+1}\right]-\mathbb{E}\left[\tilde{q}_{t}\right]\right)[1+(1-g) \varphi / \xi]+\gamma \rho\left(\mathbb{E}\left[\tilde{e}_{t+1}\right]-\mathbb{E}\left[\tilde{e}_{t}\right]\right) \\
& =r_{S}+\gamma(1-g)\left(q_{S}-\mathbb{E}\left[\tilde{q}_{t}\right]\right)[1+(1-g) \varphi / \xi]+\gamma \rho(1-\theta)\left(e_{S}-\mathbb{E}\left[\tilde{e}_{t}\right]\right)
\end{aligned}
$$

where, solving the recursion from $T$ to $t>T, \mathbb{E}\left[\tilde{q}_{t}\right]=q_{S}-g^{t-T}\left(q_{S}-q_{T}\right)$ and $\mathbb{E}\left[\tilde{e}_{t}\right]=$ $e_{S}-\theta^{t-T}\left(e_{S}-e_{T}\right)$ with $e_{T}<e_{S}=e_{N}$ along an ENP and $e_{S}=e_{T}<e_{N}$ in the case of an SEP. Substituting gives 42 . Now, from 13 and $\mathbb{E}\left[\tilde{\mu}_{t}\right]=\mathbb{E}\left[\tilde{q}_{t}\right] \varphi / \xi+(1-\theta)\left(e_{N}-e_{S}\right) / \xi$, we get

$$
z_{t}=\mathbb{E}\left[\tilde{r}_{t}\right]+(1-\lambda)\left(\mathbb{E}\left[\tilde{q}_{t+1}\right]-\mathbb{E}\left[\tilde{q}_{t}\right]\right) \varphi / \xi=\mathbb{E}\left[\tilde{r}_{t}\right]+(1-\lambda)(1-g) g^{t-T}\left(q_{S}-q_{T}\right) \varphi / \xi
$$

hence 41 . Replacing in $(37)$ and 42 yield

$Z_{t}=a_{5} \sum_{i=0}^{+\infty}\left(a_{5} \gamma\right)^{i}\left\{(1-g) g^{t+i-T}\left(q_{S}-q_{T}\right)[\gamma+(1-\lambda+\gamma(1-g)) \varphi / \xi]+\gamma \rho(1-\theta) \theta^{t+i-T}\left(e_{S}-e_{T}\right)\right\}$

which gives (43). The SEP stationary state $e_{S}$ is derived as follows. Suppose the economy has reached an SEP at date $T$ with corresponding GDP level $q_{T}$ and EQ level $e_{T}=e_{S}$. Using (43) to substitute for $Z_{t}$ in (36), we obtain

$$
\mu_{t+1}=a_{1} \mu_{t}+a_{2} e_{t}+a_{3} q_{t}+\Gamma_{t}+a_{4}+\frac{a_{5} r_{S}}{1-a_{5} \gamma}+a_{5} \tau \eta_{t}
$$


for all $t>T$, where $\Gamma_{t}=g \Gamma_{t-1}$ with

$$
\Gamma_{T} \equiv(1-g)\left(q_{S}-q_{T}\right)[\gamma+(1-\lambda+\gamma(1-g)) \varphi / \xi] /\left(1-a_{5} \gamma g\right)
$$

We can write the dynamic of the economy along the SEP as

$$
\tilde{Y}_{t}=B \tilde{Y}_{t-1}+H \tilde{\nu}_{t}
$$

where $\tilde{Y}_{t}=\left(\tilde{\mu}_{t}, \tilde{e}_{t}, \tilde{q}_{t}, \Gamma_{t}, 1\right)^{\prime}$ is a column vector,

$$
B=\left[\begin{array}{ccccc}
a_{1} & a_{2} & a_{3} & 1 & a_{4}+\frac{a_{5} r_{S}}{1-a_{5} \gamma} \\
\xi & \theta & -\varphi & 0 & \hat{e} \\
0 & 0 & g & 0 & \hat{g} \\
0 & 0 & 0 & 0 & 1 \\
0 & 0 & 0 & g & 0
\end{array}\right], H=\left[\begin{array}{cc}
a_{5} \tau \sigma_{\eta} & 0 \\
0 & 0 \\
0 & \sigma_{\kappa} \\
0 & 0 \\
0 & 0
\end{array}\right]
$$

and $\tilde{\nu}_{t}=\left(\tilde{\nu}_{1 t}, \tilde{\nu}_{2 t}\right)^{\prime}$ is a column vector of independent standardized Gaussian variables.

We have $\mathbb{E}\left[\tilde{Y}_{t}\right]=B \mathbb{E}\left[\tilde{Y}_{t-1}\right]$ which gives

$$
\tilde{Y}_{t}-\mathbb{E}\left[\tilde{Y}_{t}\right]=B\left(\tilde{Y}_{t-1}-\mathbb{E}\left[\tilde{Y}_{t-1}\right]\right)+H \tilde{\nu}_{t}
$$

The covariance matrix thus satisfies

$$
\mathbb{E}\left[\left(\tilde{Y}_{t}-\mathbb{E}\left[\tilde{Y}_{t}\right]\right)\left(\tilde{Y}_{t}-\mathbb{E}\left[\tilde{Y}_{t}\right]\right)^{\prime}\right]=B\left[\mathbb{E}\left(\tilde{Y}_{t-1}-\mathbb{E}\left[\tilde{Y}_{t-1}\right]\right)\left(\tilde{Y}_{t-1}-\mathbb{E}\left[\tilde{Y}_{t-1}\right]\right)^{\prime}\right] B^{\prime}+H H^{\prime}
$$

By definition of an SEP, we have $\mathbb{E}\left[\tilde{e}_{t}\right]=e_{S}$ and $\mathbb{E}\left[\tilde{\iota}_{t} \tilde{q}_{t}\right]=\varphi \mathbb{E}\left[\tilde{q}_{t}\right]-\xi \mathbb{E}\left[\tilde{\mu}_{t}\right]=$ $(1-\theta)\left(e_{N}-e_{S}\right)$ for all $t$. As $q_{t}$ converges to its stationary value $q_{S}$, the stationary value of the AGT index is given by $\mu_{S} \equiv q_{S} \varphi / \xi+(1-\theta)\left(e_{N}-e_{S}\right) / \xi$. Hence, the stationary value of $\tilde{Y}_{t}$ is given by $Y_{S}=\left(\mu_{S}, e_{S}, q_{S}, 0,1\right)^{\prime}$ and satisfies $Y_{S}=B Y_{S}$. The stationary covariance matrix satisfies

$$
\mathbb{V}_{S} \equiv \mathbb{E}\left[\left(\tilde{Y}_{t}-Y_{S}\right)\left(\tilde{Y}_{t}-Y_{S}\right)^{\prime}\right]=B \mathbb{E}\left[\left(\tilde{Y}_{t-1}-Y_{S}\right)\left(\tilde{Y}_{t-1}-Y_{S}\right)^{\prime}\right] B^{\prime}+H H^{\prime}
$$

and thus solves the Lyapunov equation $\mathbb{V}_{S}=B \mathbb{V}_{S} B^{\prime}+H H^{\prime}$. As neither $B$ nor $H$ depend on $e_{S}$ and $q_{T}, \mathbb{V}_{S}$ is independent of the date at which the SEP is reached (it only depends on the parameters of the model). As the dynamic (74) is linear, the distribution of $\tilde{Y}_{t}$ follows a Gaussian distribution with mean $Y_{S}$ and covariance matrix $\mathbb{V}_{S}$ at the stationary equilibrium. Denoting by $\sigma_{e}$ the standard deviation corresponding to EQ, its 
stationary distribution must satisfy $\alpha=\operatorname{Pr}\left\{\tilde{e}_{t} \leq \bar{e}\right\}=\operatorname{Pr}\left\{\left(\tilde{e}_{t}-e_{S}\right) / \sigma_{e} \leq\left(\bar{e}-e_{S}\right) / \sigma_{e}\right\}$.

Denoting by $\Phi(x)$ the CDF of the standardized Gaussian variable at level $x$ and using $\Phi(-x)=1-\Phi(x)$, we get $\alpha=\operatorname{Pr}\left\{\tilde{e}_{t} \leq \bar{e}\right\}=1-\Phi\left(\left(e_{S}-\bar{e}\right) / \sigma_{e}\right)$, hence $e_{S}=$ $\bar{e}+\sigma_{e} \Phi^{-1}(1-\alpha)$.

The covariance term in $(27)$ is derived using $u_{\phi}\left(y_{t}\right)=-e^{-\gamma y_{t}}$ and $\mathbb{E}\left[e^{-\gamma \tilde{y}}\right]=$ $e^{-\gamma(\mathbb{E}[\tilde{y}]-\gamma \mathbb{V}[\tilde{y}] / 2)}$ which gives

$$
\frac{u_{\phi}^{\prime}\left(\tilde{y}_{t+1}\right)}{\mathbb{E}_{t-1}\left[u_{\phi}^{\prime}\left(\tilde{y}_{t+1}\right)\right]}=e^{-\gamma\left(\tilde{y}_{t+1}-\mathbb{E}_{t-1}\left[\tilde{y}_{t+1}\right]+\gamma \sigma_{y_{+1}}^{2} / 2\right)} .
$$

Consequently,

$$
\begin{array}{r}
\frac{\operatorname{Cov}_{t-1}\left(\tilde{r}_{t}, u_{\phi}^{\prime}\left(\tilde{y}_{t+1}\right)\right)}{\mathbb{E}_{t-1}\left[u_{\phi}^{\prime}\left(\tilde{y}_{t+1}\right)\right]}=\mathbb{E}\left[\left(\tilde{r}_{t}-\mathbb{E}_{t-1}\left[\tilde{r}_{t}\right]\right)\left(\frac{u_{\phi}^{\prime}\left(\tilde{y}_{t+1}\right)}{\mathbb{E}_{t-1}\left[u_{\phi}^{\prime}\left(\tilde{y}_{t+1}\right)\right]}-1\right)\right] \\
=\mathbb{E}\left[\left\{\left[1-(1-\lambda) a_{5}\right] a_{5} \tau \tilde{\eta}_{t}-(1-\lambda) a_{3} \tilde{\kappa}_{t}\right\}\left(e^{-\gamma\left(\tilde{y}_{t+1}-\mathbb{E}_{t-1}\left[\tilde{y}_{t+1}\right]+\gamma \sigma_{y_{+1}}^{2} / 2\right)}-1\right)\right] \\
=e^{-\gamma^{2} \sigma_{y_{+1}}^{2} / 2}\left\{\left[1-(1-\lambda) a_{5}\right] \mathbb{E}\left[\tau \tilde{\eta}_{t} e^{-\gamma\left(\tilde{y}_{t+1}-\mathbb{E}\left[\tilde{y}_{t+1}\right]\right)}\right]\right. \\
\left.-(1-\lambda) a_{3} \mathbb{E}\left[\tilde{\kappa}_{t} e^{-\gamma\left(\tilde{y}_{t+1}-\mathbb{E}_{t-1}\left[\tilde{y}_{t+1}\right]\right)}\right]\right\}
\end{array}
$$

where the last term can be written as

$$
\begin{aligned}
\mathbb{E}\left[\tilde{\kappa}_{t} e^{-\gamma\left(\tilde{y}_{t+1}-\mathbb{E}_{t-1}\left[\tilde{y}_{t+1}\right]\right)}\right] & =\mathbb{E}\left[\tilde{\kappa}_{t} e^{-\gamma\left[\left(1-a_{3}\right) g+\left(1-a_{1}\right) a_{3}+\left(a_{2}-\rho\right) \varphi\right] \tilde{\kappa}_{t}}\right] \\
& \times \mathbb{E}\left[e^{-\gamma\left[\left(1-a_{3}\right) \tilde{\kappa}_{t+1}+\left(1-a_{1}\right) a_{5} \tau \tilde{\eta}_{t}-a_{5} \tau \tilde{\eta}_{t+1}\right]}\right.
\end{aligned}
$$

from independence. Using $\mathbb{E}\left[e^{-\gamma \tilde{X}}\right]=e^{-\gamma\left(\mathbb{E} \tilde{X}-\gamma \sigma_{X}^{2} / 2\right)}$ for a normal random variable $\tilde{X}$, it comes that the last term is equal to $e^{\gamma^{2}\left\{\sigma_{y}^{2}+\left(1-a_{1}\right)^{2} a_{5}^{2} \tau^{2} \sigma_{\eta}^{2}\right\} / 2}$. Moreover, using

$$
\mathbb{E}\left[\tilde{X} e^{-\gamma \tilde{X}}\right]=-\frac{d}{d \gamma} \mathbb{E}\left[e^{-\gamma \tilde{X}}\right]=-\frac{d}{d \gamma} e^{-\gamma\left(\mathbb{E} \tilde{X}-\gamma \sigma_{X}^{2} / 2\right)}=\left(\mathbb{E} \tilde{X}-\gamma \sigma_{X}^{2}\right) e^{-\gamma\left(\mathbb{E} \tilde{X}-\gamma \sigma_{X}^{2} / 2\right)}
$$

we get

$\mathbb{E}\left[\tilde{\kappa}_{t} e^{-\gamma\left[\left(1-a_{3}\right) g+\left(1-a_{1}\right) a_{3}\right] \tilde{\kappa}_{t}}\right]=-\gamma\left[\left(1-a_{3}\right) g+\left(1-a_{1}\right) a_{3}+\left(a_{2}-\rho\right) \varphi\right]^{2} \sigma_{\kappa}^{2} e^{\gamma^{2}\left[\left(1-a_{3}\right) g+\left(1-a_{1}\right) a_{3}\right]^{2} \sigma_{\kappa}^{2} / 2}$

which gives

$$
\mathbb{E}\left[\tilde{\kappa}_{t} e^{-\gamma\left(\tilde{y}_{t+1}-\mathbb{E}_{t-1}\left[\tilde{y}_{t+1}\right]\right)}\right]=-\gamma\left[\left(1-a_{3}\right) g+\left(1-a_{1}\right) a_{3}+\left(a_{2}-\rho\right) \varphi\right]^{2} \sigma_{\kappa}^{2} e^{\gamma^{2} \sigma_{y_{t+1}}^{2} / 2}
$$


Similarly, we have

$$
\begin{aligned}
\mathbb{E}\left[\left(\tau \tilde{\eta}_{t}\right) e^{-\gamma\left(\tilde{y}_{t+1}-\mathbb{E} \tilde{y}_{t+1}\right)}\right] & =\mathbb{E}\left[\left(\tau \tilde{\eta}_{t}\right) e^{-\gamma a_{5}\left(1-a_{1}\right)\left(\tau \tilde{\eta}_{t}\right)}\right] \\
& \times \mathbb{E}\left[e^{-\gamma\left\{\left(1-a_{3}\right) \tilde{\kappa}_{t+1}+\left[\left(1-a_{3}\right) g+\left(1-a_{1}\right) a_{3}\right] \tilde{\kappa}_{t}-a_{5}\left(\tau \tilde{\eta}_{t+1}\right)\right\}}\right] \\
& =-\gamma\left(1-a_{1}\right)^{2} a_{5}^{2} \tau^{2} \sigma_{\eta}^{2} e^{\gamma^{2} \sigma_{y_{t+1}}^{2} / 2} .
\end{aligned}
$$

Collecting terms, we get

$$
\begin{aligned}
\frac{\operatorname{cov}\left(\tilde{r}_{t}, u_{\phi}^{\prime}\left(\tilde{y}_{t+1}\right)\right)}{\mathbb{E}_{t-1}\left[u_{\phi}^{\prime}\left(\tilde{y}_{t+1}\right)\right]} & =(1-\lambda) a_{3} \gamma\left[\left(1-a_{3}\right) g+\left(1-a_{1}\right) a_{3}+\left(a_{2}-\rho\right) \varphi\right]^{2} \sigma_{\kappa}^{2} \\
& -\left[1-(1-\lambda) a_{5}\right] \gamma\left(1-a_{1}\right)^{2} a_{5}^{2} \tau^{2} \sigma_{\eta}^{2} \\
& =\gamma(1-\lambda) a_{3}\left(\sigma_{y+1}^{2}-\sigma_{y}^{2}\right)-\gamma\left[1-(1-\lambda)\left(a_{5}-a_{3}\right)\right]\left(1-a_{1}\right)^{2} a_{5}^{2} \tau^{2} \sigma_{\eta}^{2}
\end{aligned}
$$

which gives 40 . 


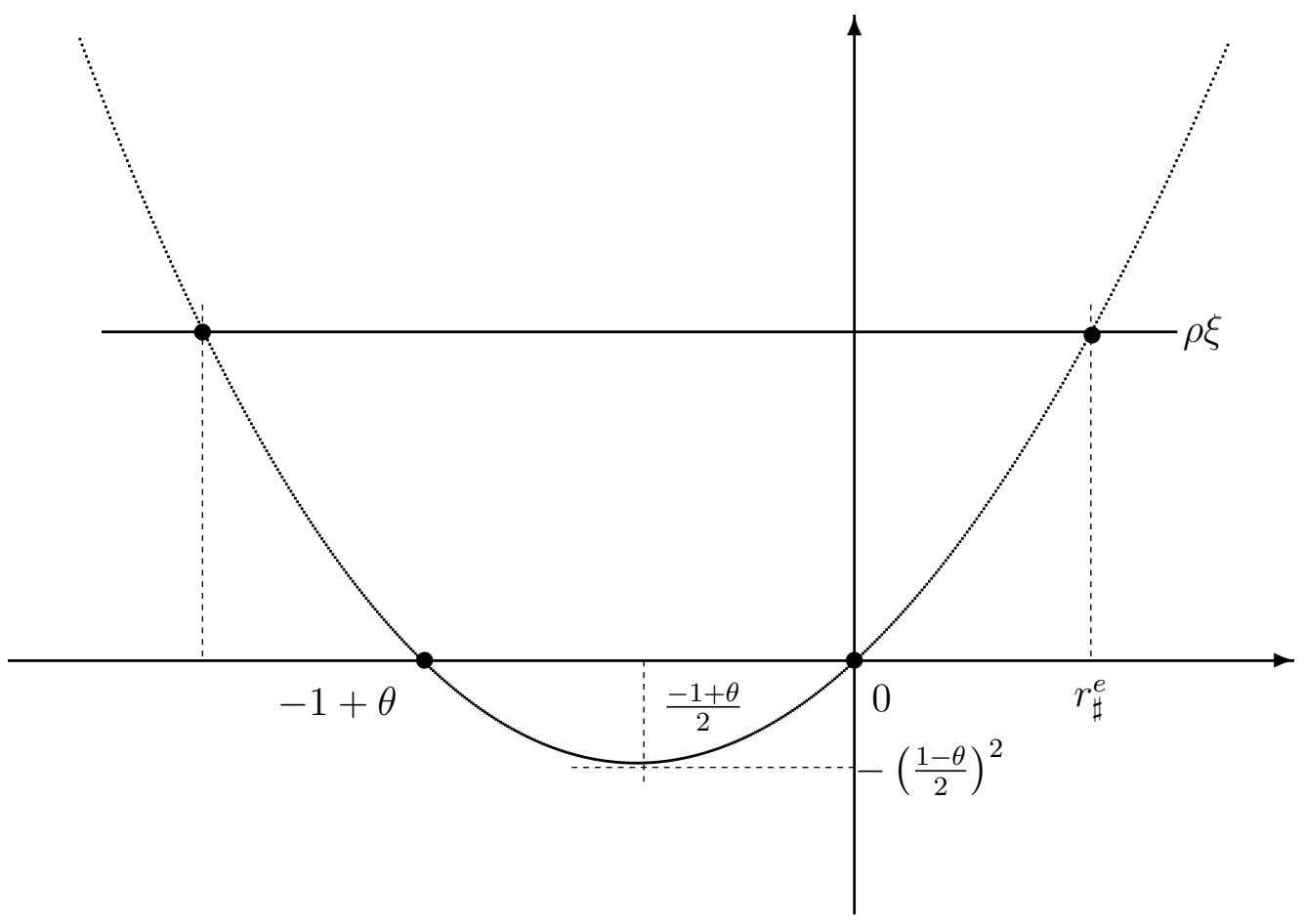

Figure 1: Long run optimal interest rate.

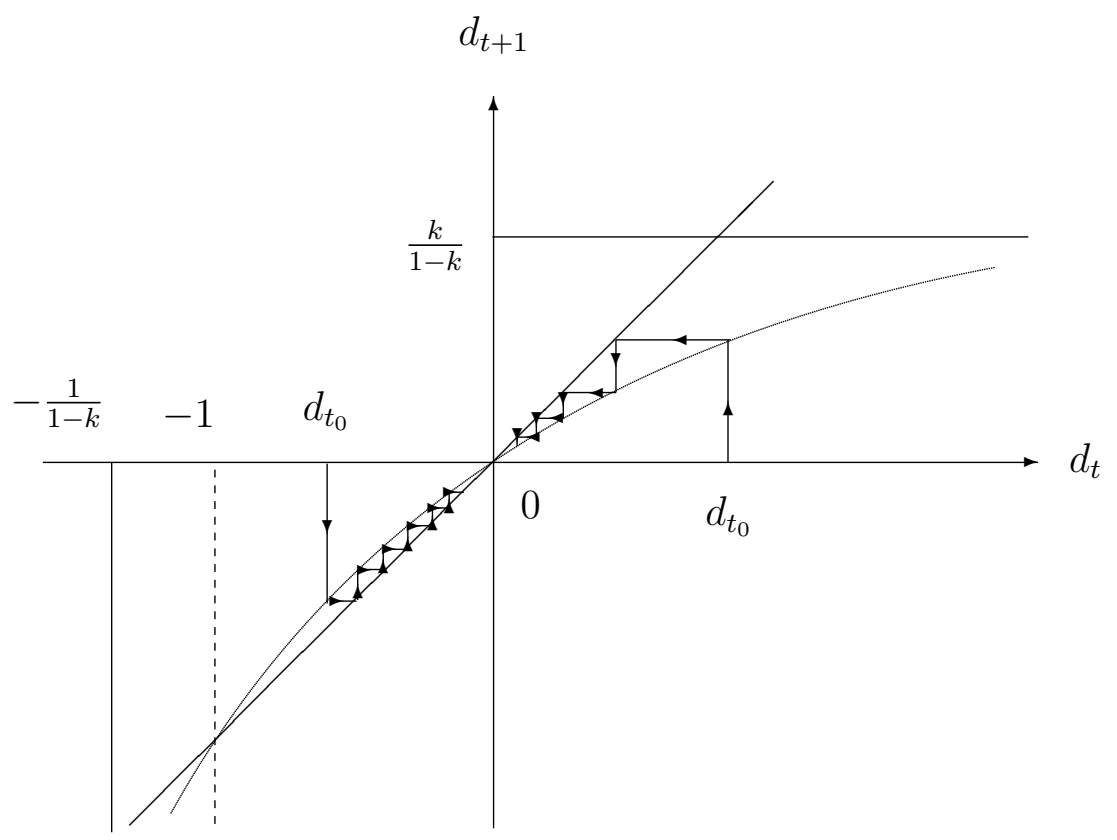

Figure 2: Convergence of the normalized gap. 


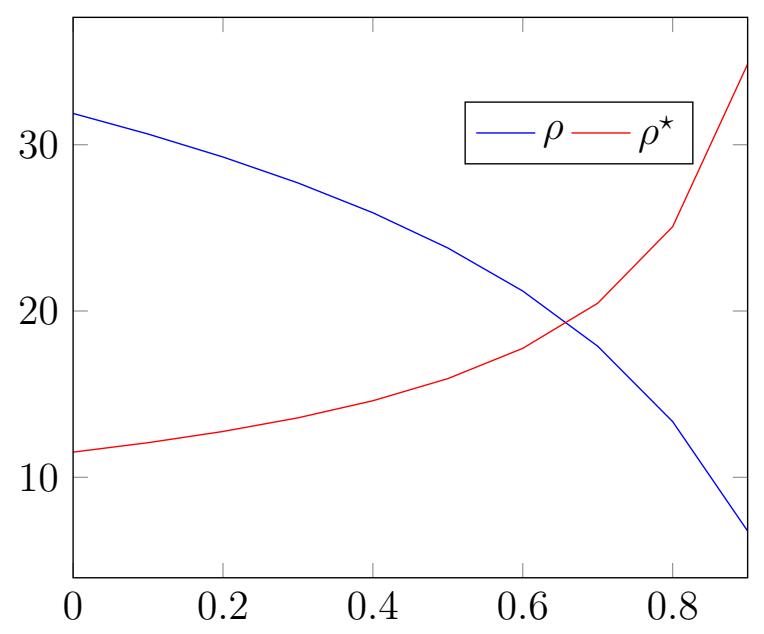

Figure 3: Carbon prices as functions of $\lambda$.

Baseline with $\lambda=.8$. Unit: US $\$ / \mathrm{t} \mathrm{CO}_{2}$

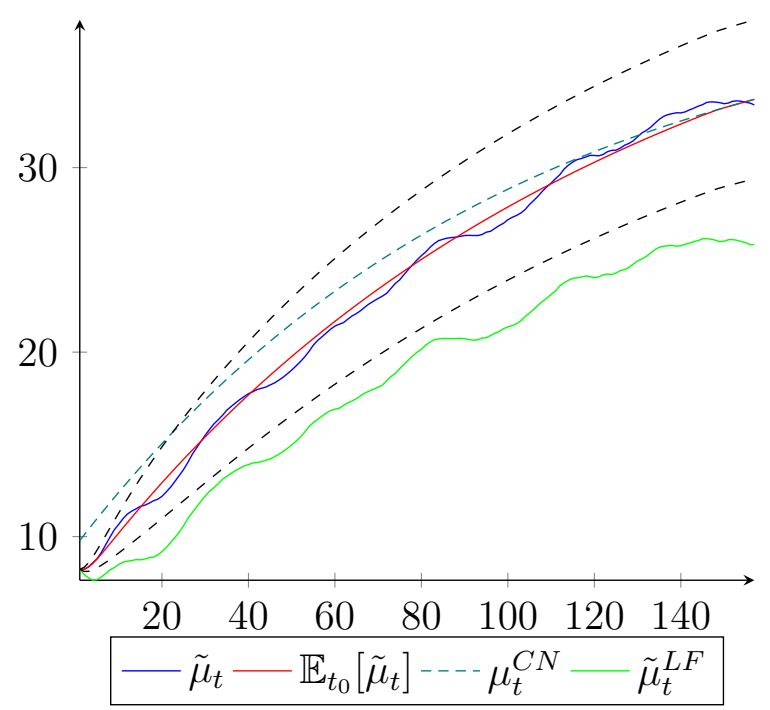

Figure 4: AGT index dynamic.

Baseline with $\lambda=.8$. 


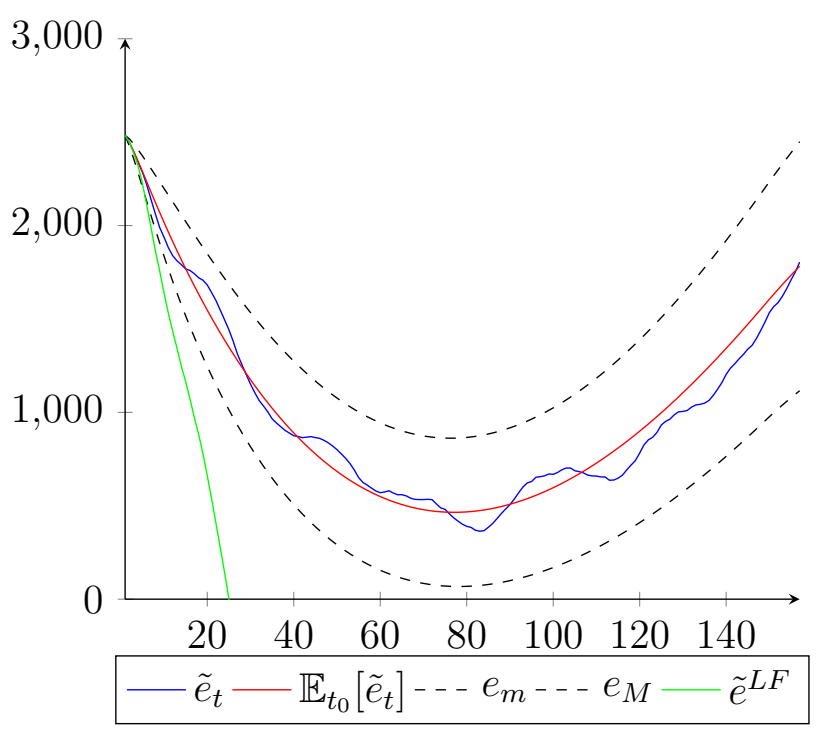

Figure 5: Environmental quality dynamic.

Baseline with $\lambda=.8$. Unit: Gt $\mathrm{CO}_{2}$

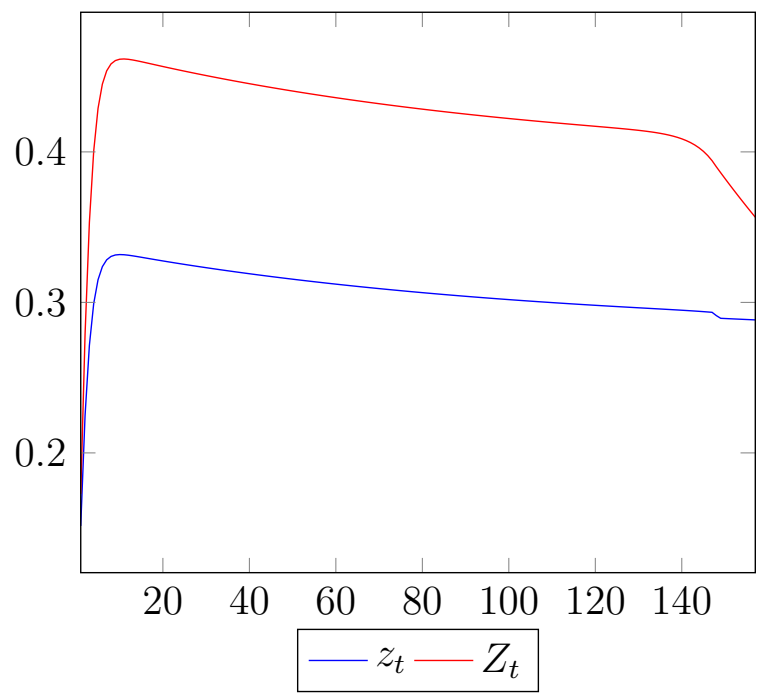

Figure 6: Optimal policy scheme.

Baseline with $\lambda=.8$. 


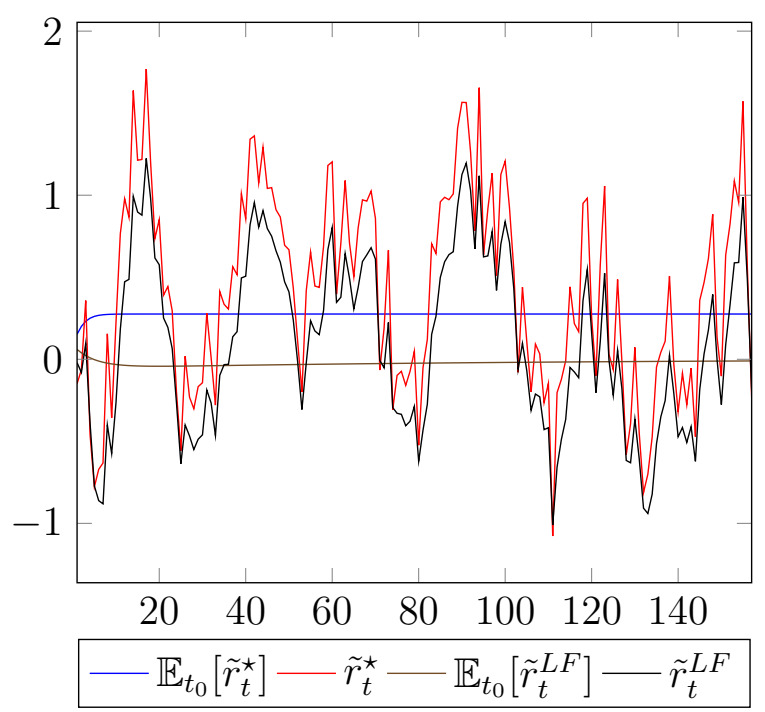

Figure 7: Interest rates.

Baseline with $\lambda=.8$.

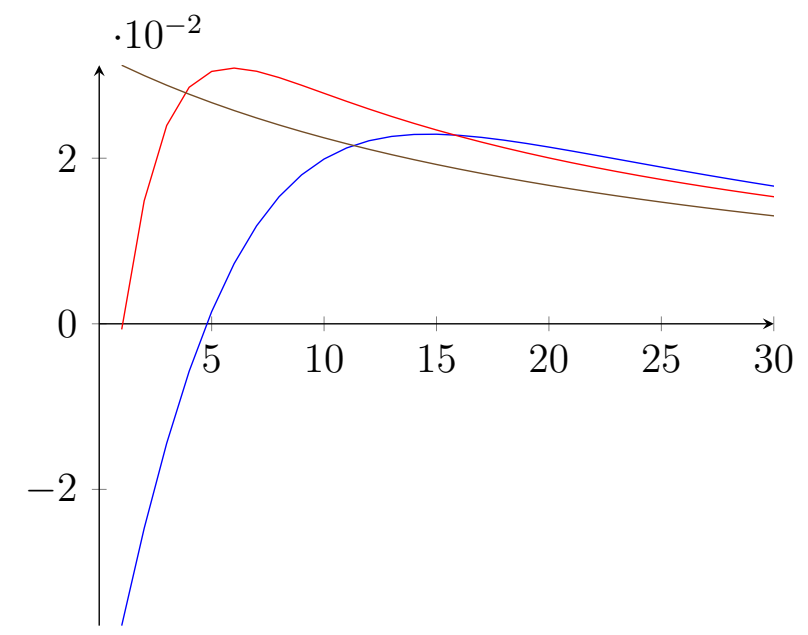

$-\mathbb{E}_{t_{0}}\left[\tilde{I}_{t}^{L F} / \tilde{\mu}_{t}^{L F}\right]-\mathbb{E}_{t_{0}}\left[\tilde{I}_{t}^{\star} / \tilde{\mu}_{t}^{\star}\right]-$ Growth rate

Figure 8: Investment rates.

Baseline with $\lambda=.8$. 


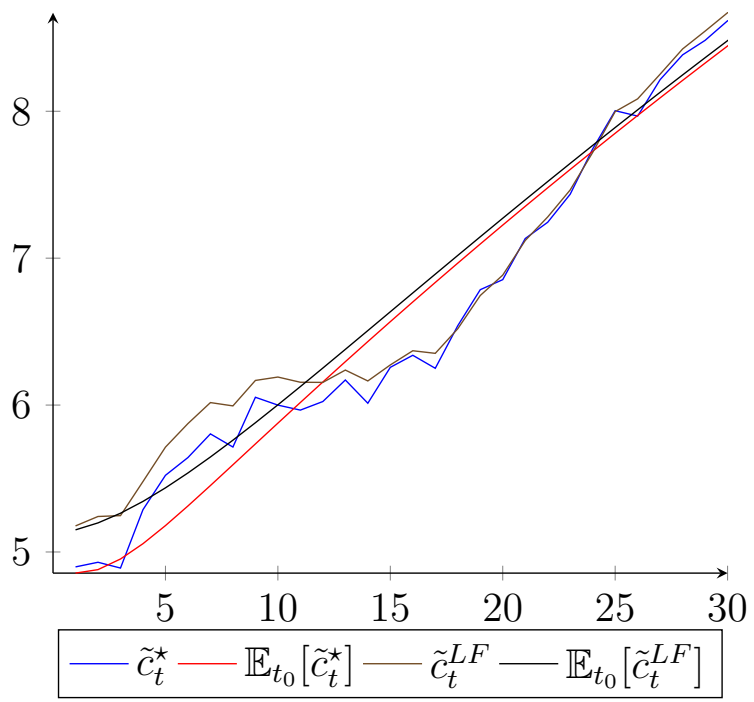

Figure 9: Consumption dynamic.

Baseline with $\lambda=.8$. Unit: 10 trillions US $\$$

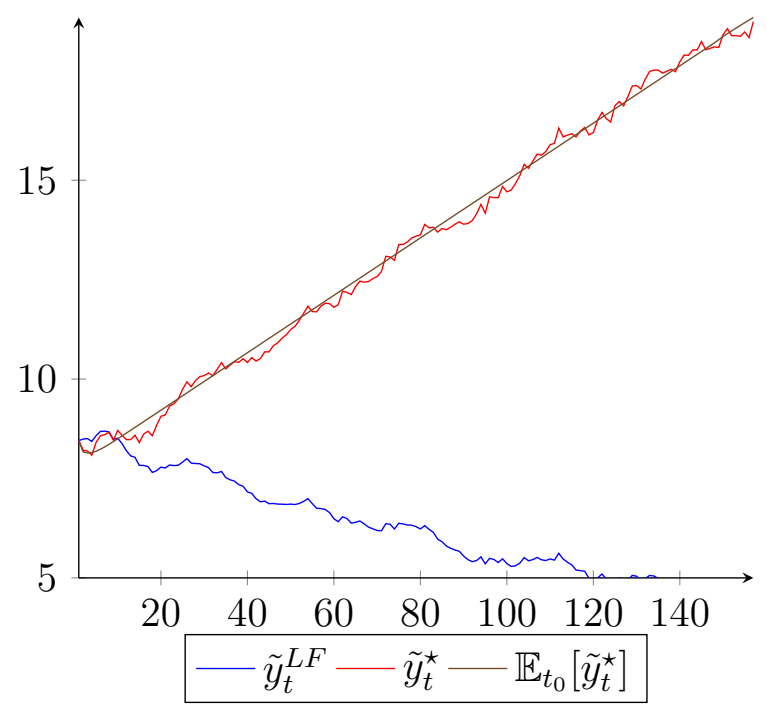

Figure 10: Total wealth dynamic.

Baseline with $\lambda=.8$. Unit: 10 trillions US\$ 
Table 1: Carbon price evaluation - Baseline

\begin{tabular}{lllllllll}
\hline$\lambda$ & $\rho$ & $\sigma_{y}$ & $\sigma_{\mu}$ & $r_{S}$ & $\rho^{\star}$ & $r_{\sharp}^{\star}$ & $T$ & $e_{T}$ \\
\hline 0 & 31.88 & $9.08 \cdot 10^{-2}$ & $5.92 \cdot 10^{-2}$ & 6.29 & 11.51 & 17.96 & 199 & 775.08 \\
.1 & 30.64 & $8.97 \cdot 10^{-2}$ & $6.03 \cdot 10^{-2}$ & 6.38 & 12.08 & 18.48 & 199 & 799.46 \\
.2 & 29.26 & $8.87 \cdot 10^{-2}$ & $6.13 \cdot 10^{-2}$ & 6.46 & 12.75 & 19.08 & 199 & 835.86 \\
.3 & 27.7 & $8.79 \cdot 10^{-2}$ & $6.21 \cdot 10^{-2}$ & 6.52 & 13.57 & 19.79 & 199 & 894.33 \\
.4 & 25.91 & $8.73 \cdot 10^{-2}$ & $6.27 \cdot 10^{-2}$ & 6.57 & 14.6 & 20.58 & 199 & 971.57 \\
.5 & 23.79 & $8.72 \cdot 10^{-2}$ & $6.28 \cdot 10^{-2}$ & 6.58 & 15.93 & 21.59 & 199 & $1,110.62$ \\
.6 & 21.2 & $8.79 \cdot 10^{-2}$ & $6.21 \cdot 10^{-2}$ & 6.52 & 17.76 & 22.91 & 199 & $1,356.75$ \\
.7 & 17.88 & $8.99 \cdot 10^{-2}$ & $6.01 \cdot 10^{-2}$ & 6.36 & 20.47 & 24.73 & 199 & $1,855.88$ \\
.8 & 13.35 & $9.47 \cdot 10^{-2}$ & $5.53 \cdot 10^{-2}$ & 5.96 & 25.08 & 27.58 & 147 & $1,541.66$ \\
.9 & 6.76 & 0.11 & $4.44 \cdot 10^{-2}$ & 4.98 & 34.85 & 32.76 & 82 & $1,046.54$ \\
\hline
\end{tabular}

Parameters: $\sigma_{\eta}=0.01, \sigma_{\kappa}=0.15, \sigma_{\epsilon}=0.01, \xi=6.5 / 2, \varphi=6.5, \gamma=3, \psi=0.1, r_{0}=$ $6 \%, \alpha=0.1 \%, \mu_{0}=8.66, \sigma_{\kappa} / q_{0}=3.19 \%$.

Table 2: Carbon price evaluation - Large GDP shocks

\begin{tabular}{lllllllll}
\hline$\lambda$ & $\rho$ & $\sigma_{y}$ & $\sigma_{\mu}$ & $r_{S}$ & $\rho^{\star}$ & $r_{\sharp}^{\star}$ & $T$ & $e_{T}$ \\
\hline 0 & 29.31 & 0.13 & $7.44 \cdot 10^{-2}$ & 2.9 & 11.18 & 17.15 & 199 & $1,189.55$ \\
.1 & 28.2 & 0.12 & $7.60 \cdot 10^{-2}$ & 3.08 & 11.68 & 17.65 & 199 & $1,216.78$ \\
.2 & 26.95 & 0.12 & $7.74 \cdot 10^{-2}$ & 3.23 & 12.27 & 18.24 & 199 & $1,262.76$ \\
.3 & 25.52 & 0.12 & $7.86 \cdot 10^{-2}$ & 3.36 & 13 & 18.92 & 199 & $1,336.38$ \\
.4 & 23.85 & 0.12 & $7.94 \cdot 10^{-2}$ & 3.45 & 13.9 & 19.68 & 199 & $1,439.4$ \\
.5 & 21.86 & 0.12 & $7.96 \cdot 10^{-2}$ & 3.47 & 15.06 & 20.65 & 199 & $1,621.95$ \\
.6 & 19.39 & 0.12 & $7.87 \cdot 10^{-2}$ & 3.38 & 16.63 & 21.87 & 199 & $1,946.53$ \\
.7 & 16.21 & 0.12 & $7.60 \cdot 10^{-2}$ & 3.07 & 18.91 & 23.52 & 166 & $1,768.81$ \\
.8 & 11.86 & 0.13 & $6.95 \cdot 10^{-2}$ & 2.34 & 22.64 & 26.01 & 123 & $1,492.76$ \\
.9 & 5.72 & 0.14 & $5.54 \cdot 10^{-2}$ & 0.59 & 29.94 & 30.24 & 68 & $1,057.43$ \\
\hline
\end{tabular}

Parameters: $\sigma_{\eta}=0.01, \sigma_{\kappa}=0.2, \sigma_{\epsilon}=0.01, \xi=6.5 / 2, \varphi=6.5, \gamma=3, \psi=$ $0.1, r_{0}=6 \%, \alpha=0.1 \%, \mu_{0}=8.66, \sigma_{\kappa} / q_{0}=4.26 \%$. 
Table 3: Carbon price evaluation - Small GDP shocks

\begin{tabular}{lllllllll}
\hline$\lambda$ & $\rho$ & $\sigma_{y}$ & $\sigma_{\mu}$ & $r_{S}$ & $\rho^{\star}$ & $r_{\sharp}^{\star}$ & $T$ & $e_{T}$ \\
\hline 0 & 33.51 & $5.92 \cdot 10^{-2}$ & $4.09 \cdot 10^{-2}$ & 8.43 & 11.37 & 18.18 & 199 & 491.92 \\
.1 & 32.19 & $5.85 \cdot 10^{-2}$ & $4.16 \cdot 10^{-2}$ & 8.46 & 11.97 & 18.68 & 199 & 504.08 \\
.2 & 30.73 & $5.78 \cdot 10^{-2}$ & $4.22 \cdot 10^{-2}$ & 8.5 & 12.69 & 19.32 & 199 & 539.44 \\
.3 & 29.09 & $5.73 \cdot 10^{-2}$ & $4.27 \cdot 10^{-2}$ & 8.52 & 13.56 & 20.01 & 199 & 578.9 \\
.4 & 27.22 & $5.69 \cdot 10^{-2}$ & $4.31 \cdot 10^{-2}$ & 8.54 & 14.66 & 20.86 & 199 & 645.85 \\
.5 & 25.02 & $5.69 \cdot 10^{-2}$ & $4.32 \cdot 10^{-2}$ & 8.54 & 16.08 & 21.91 & 199 & 756.72 \\
.6 & 22.36 & $5.73 \cdot 10^{-2}$ & $4.27 \cdot 10^{-2}$ & 8.52 & 18.06 & 23.29 & 199 & 954.69 \\
.7 & 18.95 & $5.87 \cdot 10^{-2}$ & $4.14 \cdot 10^{-2}$ & 8.45 & 21.04 & 25.23 & 199 & $1,360.06$ \\
.8 & 14.31 & $6.18 \cdot 10^{-2}$ & $3.82 \cdot 10^{-2}$ & 8.28 & 26.22 & 28.3 & 169 & $1,596.58$ \\
.9 & 7.47 & $6.92 \cdot 10^{-2}$ & $3.08 \cdot 10^{-2}$ & 7.84 & 37.74 & 34.19 & 94 & $1,036.41$ \\
\hline \\
Parameters: $\sigma_{\eta}=0.01, \sigma_{\kappa}=0.1, \sigma_{\epsilon}=0.01, \xi=6.5 / 2, \varphi=6.5, \gamma=3, \psi=0.1, r_{0}=$ \\
$6 \%, \alpha=0.1 \%, \mu_{0}=8.66, \sigma_{\kappa} / q_{0}=2.13 \%$.
\end{tabular}

Table 4: Carbon price evaluation - Highly effective GT

\begin{tabular}{lllllllll}
\hline$\lambda$ & $\rho$ & $\sigma_{y}$ & $\sigma_{\mu}$ & $r_{S}$ & $\rho^{\star}$ & $r_{\sharp}^{\star}$ & $T$ & $e_{T}$ \\
\hline 0 & 28.29 & 0.11 & $4.50 \cdot 10^{-2}$ & 5.03 & 5.35 & 17.12 & 199 & 685.81 \\
.1 & 27.01 & 0.1 & $4.53 \cdot 10^{-2}$ & 5.06 & 5.68 & 17.75 & 199 & 742.45 \\
.2 & 25.59 & 0.1 & $4.54 \cdot 10^{-2}$ & 5.08 & 6.06 & 18.46 & 199 & 821.93 \\
.3 & 23.97 & 0.1 & $4.54 \cdot 10^{-2}$ & 5.08 & 6.54 & 19.27 & 199 & 927.89 \\
.4 & 22.1 & 0.1 & $4.51 \cdot 10^{-2}$ & 5.05 & 7.14 & 20.26 & 199 & $1,091.92$ \\
.5 & 19.91 & 0.11 & $4.44 \cdot 10^{-2}$ & 4.98 & 7.93 & 21.48 & 199 & $1,355.49$ \\
.6 & 17.25 & 0.11 & $4.29 \cdot 10^{-2}$ & 4.84 & 9.03 & 23.07 & 199 & $1,821.79$ \\
.7 & 13.94 & 0.11 & $4.04 \cdot 10^{-2}$ & 4.6 & 10.68 & 25.29 & 158 & $1,569.13$ \\
.8 & 9.68 & 0.11 & $3.60 \cdot 10^{-2}$ & 4.15 & 13.48 & 28.63 & 111 & $1,218.52$ \\
.9 & 4.3 & 0.12 & $2.85 \cdot 10^{-2}$ & 3.35 & 19.16 & 34.41 & 59 & 790.66 \\
\hline Parameters: & $\sigma_{\eta}=0.01, \sigma_{\kappa}=0.15, \sigma_{\epsilon}=0.01, \xi=6.5, \varphi=6.5, \gamma=3, \psi=$ \\
$0.1, r_{0}=6 . \%, \alpha=0.1 \%, \mu_{0}=4.3, \sigma_{\kappa} / q_{0}=3.19 \%$. & \multicolumn{5}{l}{$c$}
\end{tabular}


Table 5: Carbon price evaluation - Less effective GT

\begin{tabular}{lllllllll}
\hline$\lambda$ & $\rho$ & $\sigma_{y}$ & $\sigma_{\mu}$ & $r_{S}$ & $\rho^{\star}$ & $r_{\sharp}^{\star}$ & $T$ & $e_{T}$ \\
\hline 0 & 32.88 & $8.33 \cdot 10^{-2}$ & $6.67 \cdot 10^{-2}$ & 6.88 & 20.8 & 19.96 & 199 & $1,063.11$ \\
.1 & 31.63 & $8.17 \cdot 10^{-2}$ & $6.83 \cdot 10^{-2}$ & 7 & 21.6 & 20.39 & 199 & $1,075.53$ \\
.2 & 30.25 & $8.01 \cdot 10^{-2}$ & $6.99 \cdot 10^{-2}$ & 7.11 & 22.57 & 20.92 & 199 & $1,103.45$ \\
.3 & 28.71 & $7.86 \cdot 10^{-2}$ & $7.14 \cdot 10^{-2}$ & 7.22 & 23.75 & 21.55 & 199 & $1,150.22$ \\
.4 & 26.96 & $7.73 \cdot 10^{-2}$ & $7.27 \cdot 10^{-2}$ & 7.31 & 25.24 & 22.25 & 199 & $1,213.49$ \\
.5 & 24.91 & $7.64 \cdot 10^{-2}$ & $7.36 \cdot 10^{-2}$ & 7.37 & 27.15 & 23.16 & 199 & $1,329.64$ \\
.6 & 22.44 & $7.62 \cdot 10^{-2}$ & $7.38 \cdot 10^{-2}$ & 7.39 & 29.72 & 24.32 & 199 & $1,533.17$ \\
.7 & 19.3 & $7.74 \cdot 10^{-2}$ & $7.26 \cdot 10^{-2}$ & 7.3 & 33.48 & 25.91 & 199 & $1,932.57$ \\
.8 & 14.95 & $8.16 \cdot 10^{-2}$ & $6.84 \cdot 10^{-2}$ & 7 & 39.72 & 28.39 & 154 & $1,689.05$ \\
.9 & 8.25 & $9.35 \cdot 10^{-2}$ & $5.66 \cdot 10^{-2}$ & 6.07 & 52.79 & 32.94 & 92 & $1,258.7$ \\
\hline
\end{tabular}

Parameters: $\sigma_{\eta}=0.01, \sigma_{\kappa}=0.15, \sigma_{\epsilon}=0.01, \xi=6.5 / 3, \varphi=6.5, \gamma=3, \psi=0.1, r_{0}=$ $6 . \%, \alpha=0.1 \%, \mu_{0}=12.99, \sigma_{\kappa} / q_{0}=3.19 \%$.

Table 6: Carbon price evaluation - Large emissions potential

\begin{tabular}{lllllllll}
\hline$\lambda$ & $\rho$ & $\sigma_{y}$ & $\sigma_{\mu}$ & $r_{S}$ & $\rho^{\star}$ & $r_{\sharp}^{\star}$ & $T$ & $e_{T}$ \\
\hline 0 & 28.69 & $8.92 \cdot 10^{-2}$ & $6.08 \cdot 10^{-2}$ & 6.42 & 12.2 & 20.02 & 199 & $1,007.51$ \\
.1 & 27.54 & $8.82 \cdot 10^{-2}$ & $6.18 \cdot 10^{-2}$ & 6.5 & 12.73 & 20.52 & 199 & $1,046.48$ \\
.2 & 26.27 & $8.73 \cdot 10^{-2}$ & $6.27 \cdot 10^{-2}$ & 6.57 & 13.35 & 21.11 & 199 & $1,104.98$ \\
.3 & 24.83 & $8.65 \cdot 10^{-2}$ & $6.35 \cdot 10^{-2}$ & 6.63 & 14.11 & 21.8 & 199 & $1,191.06$ \\
.4 & 23.19 & $8.61 \cdot 10^{-2}$ & $6.39 \cdot 10^{-2}$ & 6.67 & 15.07 & 22.57 & 199 & $1,303.89$ \\
.5 & 21.25 & $8.61 \cdot 10^{-2}$ & $6.39 \cdot 10^{-2}$ & 6.67 & 16.29 & 23.56 & 199 & $1,497.03$ \\
.6 & 18.89 & $8.69 \cdot 10^{-2}$ & $6.31 \cdot 10^{-2}$ & 6.6 & 17.96 & 24.83 & 199 & $1,828.48$ \\
.7 & 15.88 & $8.91 \cdot 10^{-2}$ & $6.09 \cdot 10^{-2}$ & 6.43 & 20.41 & 26.59 & 170 & $1,736.22$ \\
.8 & 11.79 & $9.42 \cdot 10^{-2}$ & $5.58 \cdot 10^{-2}$ & 6.01 & 24.5 & 29.34 & 127 & $1,456.48$ \\
.9 & 5.93 & 0.11 & $4.46 \cdot 10^{-2}$ & 4.99 & 32.94 & 34.26 & 71 & $1,001.47$ \\
\hline
\end{tabular}

Parameters: $\sigma_{\eta}=0.01, \sigma_{\kappa}=0.15, \sigma_{\epsilon}=0.01, \xi=7.5 / 2, \varphi=7.5, \gamma=3, \psi=0.1, r_{0}=$ $6 . \%, \alpha=0.1 \%, \mu_{0}=8.76, \sigma_{\kappa} / q_{0}=3.19 \%$. 
Table 7: Carbon price evaluation - Low emissions potential

\begin{tabular}{lllllllll}
\hline$\lambda$ & $\rho$ & $\sigma_{y}$ & $\sigma_{\mu}$ & $r_{S}$ & $\rho^{\star}$ & $r_{\sharp}^{\star}$ & $T$ & $e_{T}$ \\
\hline 0 & 38.28 & $9.40 \cdot 10^{-2}$ & $5.60 \cdot 10^{-2}$ & 6.02 & 10.17 & 14.53 & 199 & 546.22 \\
.1 & 36.87 & $9.28 \cdot 10^{-2}$ & $5.72 \cdot 10^{-2}$ & 6.12 & 10.79 & 15.06 & 199 & 548.52 \\
.2 & 35.3 & $9.17 \cdot 10^{-2}$ & $5.83 \cdot 10^{-2}$ & 6.22 & 11.53 & 15.67 & 199 & 558.9 \\
.3 & 33.52 & $9.07 \cdot 10^{-2}$ & $5.93 \cdot 10^{-2}$ & 6.3 & 12.43 & 16.39 & 199 & 580.72 \\
.4 & 31.45 & $9.00 \cdot 10^{-2}$ & $6.01 \cdot 10^{-2}$ & 6.36 & 13.57 & 17.2 & 199 & 610.94 \\
.5 & 28.99 & $8.96 \cdot 10^{-2}$ & $6.04 \cdot 10^{-2}$ & 6.39 & 15.06 & 18.23 & 199 & 677.41 \\
.6 & 25.97 & $9.00 \cdot 10^{-2}$ & $6.00 \cdot 10^{-2}$ & 6.36 & 17.13 & 19.58 & 199 & 808.42 \\
.7 & 22.06 & $9.16 \cdot 10^{-2}$ & $5.84 \cdot 10^{-2}$ & 6.22 & 20.29 & 21.46 & 199 & $1,098.21$ \\
.8 & 16.63 & $9.59 \cdot 10^{-2}$ & $5.42 \cdot 10^{-2}$ & 5.86 & 25.85 & 24.44 & 194 & $1,807.6$ \\
.9 & 8.59 & 0.11 & $4.39 \cdot 10^{-2}$ & 4.94 & 38.4 & 30.1 & 104 & $1,162.06$ \\
\hline \multicolumn{7}{l}{ Parameters: } & $\sigma_{\eta}=0.01, \sigma_{\kappa}=0.15, \sigma_{\epsilon}=0.01, \xi=2.5, \varphi=5, \gamma=3, \psi=0.1, r_{0}=$ \\
$6 . \%, \alpha=0.1 \%, \mu_{0}=8.44, \sigma_{\kappa} / q_{0}=3.19 \%$. & & & & &
\end{tabular}

Table 8: Carbon price evaluation - Very Large public signal shocks

\begin{tabular}{lllllllll}
\hline$\lambda$ & $\rho$ & $\sigma_{y}$ & $\sigma_{\mu}$ & $r_{S}$ & $\rho^{\star}$ & $r_{\sharp}^{\star}$ & $T$ & $e_{T}$ \\
\hline 0 & 38.28 & $9.40 \cdot 10^{-2}$ & $5.60 \cdot 10^{-2}$ & 6.02 & 10.17 & 14.53 & 199 & 546.3 \\
.1 & 36.87 & $9.28 \cdot 10^{-2}$ & $5.72 \cdot 10^{-2}$ & 6.12 & 10.79 & 15.06 & 199 & 548.61 \\
.2 & 35.3 & $9.17 \cdot 10^{-2}$ & $5.84 \cdot 10^{-2}$ & 6.21 & 11.53 & 15.67 & 199 & 559 \\
.3 & 33.52 & $9.07 \cdot 10^{-2}$ & $5.93 \cdot 10^{-2}$ & 6.3 & 12.43 & 16.39 & 199 & 580.83 \\
.4 & 31.45 & $9.00 \cdot 10^{-2}$ & $6.01 \cdot 10^{-2}$ & 6.36 & 13.57 & 17.2 & 199 & 611.07 \\
.5 & 28.99 & $8.96 \cdot 10^{-2}$ & $6.04 \cdot 10^{-2}$ & 6.38 & 15.06 & 18.23 & 199 & 677.55 \\
.6 & 25.97 & $9.00 \cdot 10^{-2}$ & $6.01 \cdot 10^{-2}$ & 6.35 & 17.13 & 19.58 & 199 & 808.61 \\
.7 & 22.05 & $9.16 \cdot 10^{-2}$ & $5.84 \cdot 10^{-2}$ & 6.22 & 20.29 & 21.46 & 199 & $1,098.46$ \\
.8 & 16.63 & $9.59 \cdot 10^{-2}$ & $5.42 \cdot 10^{-2}$ & 5.86 & 25.85 & 24.44 & 194 & $1,808.01$ \\
.9 & 8.58 & 0.11 & $4.40 \cdot 10^{-2}$ & 4.93 & 38.39 & 30.09 & 104 & $1,162.39$ \\
\hline
\end{tabular}

Parameters: $\sigma_{\eta}=1, \sigma_{\kappa}=0.15, \sigma_{\epsilon}=0.01, \xi=6.5 / 2, \varphi=6.5, \gamma=3, \psi=0.1, r_{0}=$ $6 \%, \alpha=0.1 \%, \mu_{0}=8.66, \sigma_{\kappa} / q_{0}=3.19 \%$. 